\title{
SN 2011fu: a type IIb supernova with a luminous double-peaked light curve
}

\author{
A. Morales-Garoffolo, ${ }^{1 \star}$ N. Elias-Rosa, ${ }^{2 \star}$ M. Bersten,,${ }^{3,4,5}$ A. Jerkstrand, ${ }^{6}$ \\ S. Taubenberger, ${ }^{7,8}$ S. Benetti, ${ }^{2}$ E. Cappellaro, ${ }^{2}$ R. Kotak, ${ }^{6}$ A. Pastorello, ${ }^{2}$ F. Bufano, ${ }^{9}$ \\ R. M. Domínguez, ${ }^{10}$ M. Ergon, ${ }^{11}$ M. Fraser, ${ }^{12}$ X. Gao, ${ }^{13}$ E. García,${ }^{14,15,16}$ \\ D. A. Howell, ${ }^{17,18}$ J. Isern, ${ }^{1}$ S. J. Smartt, ${ }^{6}$ L. Tomasella ${ }^{2}$ and S. Valenti ${ }^{17,18}$ \\ ${ }^{1}$ Institut de Ciències de l'Espai (CSIC-IEEC), Campus UAB, Camí de Can Magrans S/N, E-08193 Cerdanyola (Barcelona), Spain \\ ${ }^{2}$ INAF-Osservatorio Astronomico di Padova, Vicolo dell'Osservatorio 5, I-35122 Padova, Italy \\ ${ }^{3}$ Instituto de Astrofísica La Plata, IALP (CCT La Plata), CON ICET-UNLP, Paseo del Bosque s/n, 1900 La Plata, Argentina \\ ${ }^{4}$ Facultad de Ciencias Astronómicas y Geofísicas, Universidad Nacional de La Plata, Paseo del Bosque s/n, 1900 La Plata, Argentina \\ ${ }^{5}$ Kavli Institute for the Physics and Mathematics of the Universe (WPI), The University of Tokyo, Kashiwa, Chiba 277-8583, Japan \\ ${ }^{6}$ Astrophysics Research Centre, School of Mathematics and Physics, Queen's University Belfast, Belfast BT7 1NN, UK \\ ${ }^{7}$ Max-Planck-Institut für Astrophysik, Karl-Schwarzschild-Str. 1, D-85741 Garching bei München, Germany \\ ${ }^{8}$ European Southern Observatory, Karl-Schwarzschid-Str 2, D-85748 Garching bei München, Germany \\ ${ }^{9}$ INAF-Osservatorio Astrofisico di Catania, Via S.Sofia 78, I-95123, Catania, Italy \\ ${ }^{10}$ Instituto de Física de Cantabria (IFCA), Observatorio Astronómico de Cantabria, Av. de los Castros, E-39005 Santander (Cantabria), Spain \\ ${ }^{11}$ The Oskar Klein Centre, Department of Astronomy, Stockhom University, Albanova, SE-10691 Stockholm, Sweden \\ ${ }^{12}$ Institute of Astronomy, University of Cambridge, Madingley Road, Cambridge CB3 OHA, UK \\ ${ }^{13}$ No.1 Senior High School, Urumqi, 830002 Xinjiang, China \\ ${ }^{14}$ Departamento de Física Aplicada I, E.T.S. Ingeniería, Universidad del País Vasco, 9 Alameda Urquijo s/n, E-48013 Bilbao, Spain \\ ${ }^{15}$ Esteve Duran Observatory, Foundation Observatori Esteve Duran. Avda. Montseny 46, E-Seva 08553, Spain \\ ${ }^{16}$ Institut d'Estudis Espacials de Catalunya (IEEC), Edif. Nexus, C/ Gran Capità 2-4, E-08034 Barcelona, Spain \\ ${ }^{17}$ Las Cumbres Observatory Global Telescope Network, 6740 Cortona Dr., Suite 102, Goleta, CA 93117, USA \\ ${ }^{18}$ Department of Physics, University of California, Santa Barbara, Broida Hall, Mail Code 9530, Santa Barbara, CA 93196-9530, USA
}

\begin{abstract}
We present optical and near-infrared observations of the type IIb supernova (SN) 2011fu from a few days to $\sim 300 \mathrm{~d}$ after explosion. The SN presents a double-peaked light curve (LC) similar to that of SN 1993J, although more luminous and with a longer cooling phase after the primary peak. The spectral evolution is also similar to SN 1993J's, with hydrogen dominating the spectra to $\sim 40 \mathrm{~d}$, then helium gaining strength, and nebular emission lines appearing from $\sim 60 \mathrm{~d}$ post-explosion. The velocities derived from the P-Cygni absorptions are overall similar to those of other type IIb SNe. We have found a strong similarity between the oxygen and magnesium line profiles at late times, which suggests that these lines are forming at the same location within the ejecta. The hydrodynamical modelling of the pseudo-bolometric LC and the observed photospheric velocities suggest that SN 2011fu was the explosion of an extended star $\left(R \sim 450 \mathrm{R}_{\odot}\right)$, in which $1.3 \times 10^{51} \mathrm{erg}$ of kinetic energy were released and $0.15 \mathrm{M}_{\odot}$ of ${ }^{56} \mathrm{Ni}$ were synthesized. In addition, a better reproduction of the observed early pseudo-bolometric LC is achieved if a more massive $\mathrm{H}$-rich envelope than for other type $\mathrm{IIb}$ $\mathrm{SNe}$ is considered $\left(0.3 \mathrm{M}_{\odot}\right)$. The hydrodynamical modelling of the $\mathrm{LC}$ and the comparison of our late-time spectra with nebular spectral models for type IIb SNe, point to a progenitor for SN 2011fu with a Zero Age Main Sequence (ZAMS) mass of 13-18 $\mathrm{M}_{\odot}$.
\end{abstract}

Key words: supernovae: general-supernovae: individual: SN $2011 \mathrm{fu}$. 
( $M_{\text {ZAMS }}>8 \mathrm{M}_{\odot}$; see e.g. Heger et al. 2003). They are spectroscopically divided in different groups according mainly to the presence of the $\mathrm{H}$ and $\mathrm{He}$ lines in their optical spectra. While type II SNe show prominent $\mathrm{H}$ features, type I do not, and can be further subdivided, according to the presence or absence of $\mathrm{He}$, as types $\mathrm{Ib}$ and Ic, respectively. There are also hybrid objects which undergo a transition from being $\mathrm{H}$ dominated at early phases of their spectral evolution, to He dominated at later times. These are termed type IIb SNe. The first SN to have shown this spectral transition was SN 1987K, and its progenitor was proposed to be a star that had lost most of its hydrogen envelope before exploding (Filippenko et al. 1988). The best-studied type IIb SN to date is SN 1993J (e.g. Nomoto et al. 1993; Podsiadlowski et al. 1993; Woosley et al. 1994), that is considered the prototype of the subclass. Its light curve (LC) showed an early peak, followed by a rapid decline thought to be the consequence of the cooling of the progenitor's stellar envelope after shock breakout. Subsequently, it presented a secondary maximum attributed to input from the radioactive decay of ${ }^{56} \mathrm{Ni}$. The star that exploded as SN 1993J was detected in archival images (Aldering, Humphreys \& Richmond 1994), and its disappearance was confirmed by Maund \& Smartt (2009). Two mechanisms have been proposed by which type IIb SN progenitors lose part of their $\mathrm{H}$ envelope before exploding, namely stripping by a close companion after Roche lobe overflow, and wind-driven stellar mass loss in a single star. The former scenario has gained strength over the past years, for example with the detection of signatures of the possible hot blue companion star of SN 1993J (Maund et al. 2004; see also Fox et al. 2014), and the modelling of a progenitor binary system and likely detection of the stellar companion of SN 2011dh (Benvenuto, Bersten \& Nomoto 2013; Folatelli et al. 2014). Moreover, although a Wolf-Rayet (WR) wind-like spectrum was obtained for the type IIb SN 2013cu a few hours after explosion (Gal-Yam et al. 2014), recent work has shown that the progenitor star for SN 2013cu was unlikely a WR (Groh 2014; Shivvers et al. 2015; Smith et al. 2015).

An important question is which of the observed properties of $\mathrm{SNe}$ IIb can be connected with the characteristics of their progenitor systems. For example, Chevalier \& Soderberg (2010) proposed that bright early luminosity from the shock-heated progenitor stellar envelope, low radio shell velocities, and thermal X-ray emission were characteristic of extended progenitors with $R \sim 150 \mathrm{R}_{\odot}$, while compact progenitors with $R \sim 1 \mathrm{R}_{\odot}$ have faint early optical LCs, have high radio shell velocities and non-thermal X-ray emission. However, this link is not straightforward and needs to be revised since the type IIb SNe 2011dh and 2011hs present fast expanding radio shells but the hydrodynamical modelling of their LC (Bersten et al. 2012; Bufano et al. 2014), and in the case of SN 2011dh the detection of its progenitor in archival images (Maund et al. 2011; Van Dyk et al. 2011) and its disappearance (Van Dyk et al. 2013), point to the explosion of extended stars. To further complicate the picture, $\mathrm{SNe}$ IIb show a variety of continuum flux excess in early UV spectra (Ben-Ami et al. 2014). Strong UV-excess suggests significant interaction between the $\mathrm{SN}$ ejecta and circumstellar material.

To date, few type IIb SNe have been thoroughly studied since they are relatively rare events. Li et al. (2011) estimated the fraction of type IIb over a volume-limited sample of 81 type II SNe to be 11.9 per cent $_{-3.6}^{+3.9}$. In this paper, we present optical and near-infrared (NIR) data for the type IIb SN 2011fu. SN $2011 \mathrm{fu}$, with coordinates $\alpha=02^{\mathrm{h}} 08^{\mathrm{m}} 21^{\mathrm{s}} .26$ and $\delta=41^{\circ} 29^{\prime} 9^{\prime \prime} .9$ (J2000), was discovered in a spiral arm of the galaxy UGC 1626 by F. Ciabattari and E. Mazzoni of the Italian Supernovae Search Project (ISSP), ${ }^{1}$ on 2011 September 21.04 uT and classified by Tomasella et al. (2011) as a young type II SN 2011 September 23.84 UT. A previous analysis of optical data of SN 2011fu was presented by Kumar et al. (2013), which confirmed it was a type IIb SN. In this manuscript, we contribute with data probing the whole SN evolution. In Section 2, we discuss the distance, reddening, and explosion epoch of SN 2011fu. In Section 3, we summarize our observations and the reduction process of the data. In Section 4, we present the optical and NIR LCs of the object, while in Section 5 we present and discuss the spectral evolution. In Section 6, we discuss the results obtained from the hydrodynamical modelling of the pseudo-bolometric LC and we compare our nebular spectra with published models for late-time type IIb SN spectra. Finally, in Section 7, we present the conclusions of our work.

\section{DISTANCE, REDDENING, AND EXPLOSION EPOCH}

The rise to a first maximum in the $V$ and $R$ LCs (see the inset of Fig. 2) implies that SN 2011 fu was discovered very soon after explosion. In fact, models show that the evolution of the SN LC during this rise should be very fast, and lasts $\sim 1 \mathrm{~d}$ for a number of configurations (e.g. Blinnikov et al. 1998; Bersten et al. 2012). By adopting $\mathrm{JD}_{\text {explo }}=2455824.5 \pm 0.7$ as the explosion date of SN $2011 \mathrm{fu}$, we obtain the best fit of the early phase of the pseudo-bolometric LC with the hydrodynamical models presented in Section 6.1. For this reason, we have chosen $\mathrm{JD}_{\text {explo }}=2455824.5 \pm 0.7$ as the explosion date of SN 2011fu, which is in agreement with the discovery epoch, the last non-detection of the object which was 2011 August 10 (JD = 2455783.5), the pre-discovery image taken by Xing Gao 2011 September 20 (JD = 2455825.2), and the classification as a young object. Note that this assumed explosion date also agrees, within the uncertainties, with the explosion epoch adopted by Kumar et al. (2013). All phases in the rest of this manuscript are given with respect to $\mathrm{JD}_{\text {explo }}=2455824.5 \pm 0.7$.

UGC 1626 is an SAB(rs)c type galaxy whose recessional velocity given by the NASA/IPAC Extragalactic Database (NED) is $5543 \pm 11 \mathrm{~km} \mathrm{~s}^{-1}$. We have performed measurements of the SN redshift from the narrow $\mathrm{H} \alpha$ emissions in its spectra and have obtained the same redshift as for its host galaxy, which we adopt in the rest of this paper. The redshift derived distance to UGC 1626, also provided by NED assuming $H_{0}=73 \pm 5 \mathrm{~km} \mathrm{~s}^{-1} \mathrm{Mpc}^{-1}, \Omega_{\mathrm{m}}=0.27$, $\Omega_{\mathrm{v}}=0.73$, and accounting for the Virgo, Great Attractor and Shapley infalls is $74.5 \pm 5.2 \mathrm{Mpc}$, i.e. $\mu=34.36 \pm 0.15 \mathrm{mag}$ (Mould et al. 2000).

The reddening in the line of sight of UGC 1626 due to the Milky Way is $E(B-V)_{\mathrm{MW}}=0.068 \pm 0.002 \mathrm{mag}$ (Schlafly \& Finkbeiner 2011). We detect a narrow absorption likely due $\mathrm{Na}$ ID $\lambda \lambda 5890$, 5896 at the host galaxy redshift. The equivalent width of this line is $0.33 \pm 0.03 \AA$ that using the relations for unresolved $\mathrm{Na}$ ID given by Poznanski, Prochaska \& Bloom (2012), provides an estimate of the reddening in the host galaxy as: $E(B-V)_{\text {host }}=0.035_{-0.029}^{+0.042}$ mag. In fact, a similar value of reddening due to the host galaxy is obtained using the relations of Turatto, Benetti \& Cappellaro (2003). In the rest of this manuscript, we adopt $E(B-V)_{\text {total }}=0.10_{-0.03}^{+0.04}$ mag as the total reddening towards SN 2011fu. We note that in Kumar et al. (2013) the relations of Munari \& Zwitter (1997) were used to

\footnotetext{
${ }^{1}$ http://italiansupernovae.org/en.html
} 
estimate the contribution to the reddening due to the host galaxy, and they obtained $E(B-V)_{\text {total }}=0.22 \pm 0.11$, which is consistent with the value we have adopted within the uncertainties.

\section{OBSERVATIONAL DATA, REDUCTION, AND CALIBRATION PROCESS}

The observational follow-up data of SN 2011 fu presented in this paper cover the period between a few days post-discovery, until the SN disappeared behind the Sun (end of 2012 February). After that, one more spectrum and one epoch of gri photometric data were obtained well into the nebular phase of the SN, 2012 July 20 UT. Thanks to a large collaboration between many European institutions, optical photometry and spectroscopy, as well as some NIR photometric data of SN 2011fu, have been collected at several sites. Some amateur data taken by F. Ciabattari and E. Mazzoni from Italy, and Xing Gao from China were also included in our analysis. A summary of the characteristics of the instruments used to acquire our photometric data can be seen in Table A1.

The list with our spectroscopic observations and the instrumental configuration used to acquire the data is presented in Table A2.

All the data were corrected for overscan, bias, and flat-field within the $\mathrm{IRAF}^{2}$ environment, except for data obtained at the Liverpool Telescope (LT) which were automatically reduced with the instrument specific pipeline. The instrumental magnitudes of the $\mathrm{SN}$ were derived via point-spread function (PSF) fitting which was done with SNOOPY (Cappellaro 2014). In the case of the $U$ and $u$ photometry, the measurements were done after performing template subtraction. For all other bands, PSF photometry at late time was verified by performing template subtractions and no major differences were found. ${ }^{3}$ A set of 15 local stars in the SN field (Fig. 1) were used to trace the photometric calibration. The stellar sequence was calibrated with the zero-points and colour terms derived for each site thanks to observations of Landolt standards (Tables B4 and B5) and the SN magnitudes were calculated relative to these. The errors of the magnitudes of the stellar sequence were obtained as the rms deviation with respect to their mean values. Given the fact that several instruments with different passbands were used to acquire our data, we performed S-corrections of our BVrRiI photometry to the Bessell system (Stritzinger et al. 2002; Pignata et al. 2004). In order to derive the S-correction terms, we used our spectral sequence of the SN. Note that we were not able to perform S-corrections to the $U-, u$-, and $z$-band photometry given the fact that our spectra do not cover completely these passbands. The SN magnitudes together with their errors, calculated by adding in quadrature the uncertainties associated with the calibration and the PSF fit, ${ }^{4}$ are reported in Tables B1-B3.

The unfiltered photometry provided by F. Ciabattari was rescaled to the $R$ band considering that the detector sensitivity peaks at this wavelength. With the same argument the data provided by X. Gao were rescaled to $V$.

\footnotetext{
${ }^{2}$ Image reduction and Analysis Facility, a software system distributed by the National Optical Astronomy Observatories (NOAO).

${ }^{3}$ The UBVRI band templates were obtained on 2012 October 23 with AFOSC at the $1.82 \mathrm{~m}$ telescope in the Asiago Observatory, while the uri band templates were obtained on 2014 October 25 at the LT with IO:O in the Roque de los Muchachos Observatory.

${ }^{4}$ Obtained by placing artificial stars with magnitudes similar to those of the $\mathrm{SN}$ at positions near the $\mathrm{SN}$ and calculating the standard deviation of their recovered magnitudes.
}

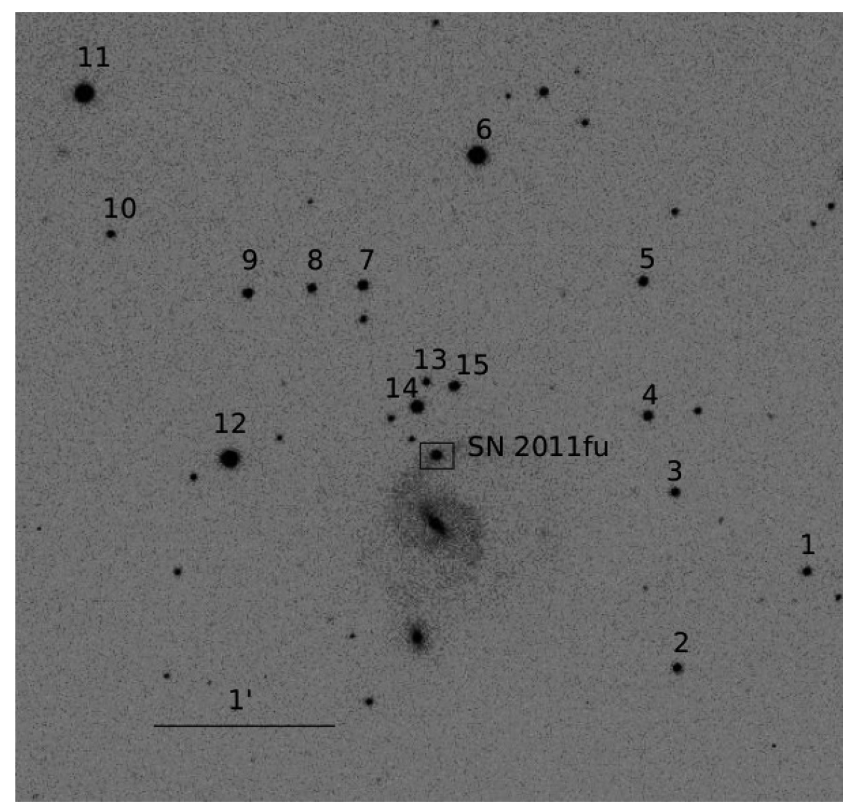

Figure 1. $V$-band image of UGC 1626 taken with the $2.2 \mathrm{~m} \mathrm{Calar}$ Alto Telescope + CAFOS on 2011 October 16. The stars used for the photometric calibration of SN 2011fu are labelled. North is to the top and east to the left.

Note that stars 2, 6, 9, 12, and 14 from our sequence coincide with stars 2, 1, 4, 3, and 5 used for photometric calibration by Kumar et al. (2013). The greatest difference between the two sequences is 0.1 mag found for star 2 in the $U$ band. For the rest of the bands differences are found to be less than $0.1 \mathrm{mag}$.

The NIR data were reduced within IRAF with the help of CAINDR, a package developed by José A. Acosta Pulido and Rafael Barrena for the instrument CAIN3 at the Telescopio Carlos Sánchez (TCS). The images of each specific filter were corrected for flat-field, and bad pixel corrections were applied. Then background images were constructed and subtracted from the science frames, and finally the images were combined taking into account the dithering pattern. Similarly to the optical data, we measured the instrumental SN magnitudes by PSF fitting. The SN calibration was done relative to 2MASS stars in the field (Skrutskie et al. 2006; see Table B4).

The spectra were corrected for overscan, bias, and flat-field within IRAF. Next they were variance-weighted extracted and wavelength calibrated with the use of arc lamp spectra. The wavelength calibration was cross-checked with respect to sky emission lines. Finally, a flux calibration was applied using sensitivity functions obtained from the observation of spectrophotometric standards. The flux calibration was cross-checked with the photometry of the SN at the nearest epoch. Telluric lines were corrected dividing the SN spectra by telluric line spectra obtained from the spectrophotometric standards.

\section{PHOTOMETRY}

\subsection{Light curves}

The optical-NIR LCs of SN 2011 fu are presented in Fig. 2. In addition to the data presented in the figure, we obtained two epochs of Sloan $g$-band data, which are listed in Table B2. In the optical, the LCs clearly present two peaks in all bands, but not in the NIR since the data do not cover the early phases. The rise to primary peak in the $V$ and $R$ bands can be seen thanks to the early-time data 


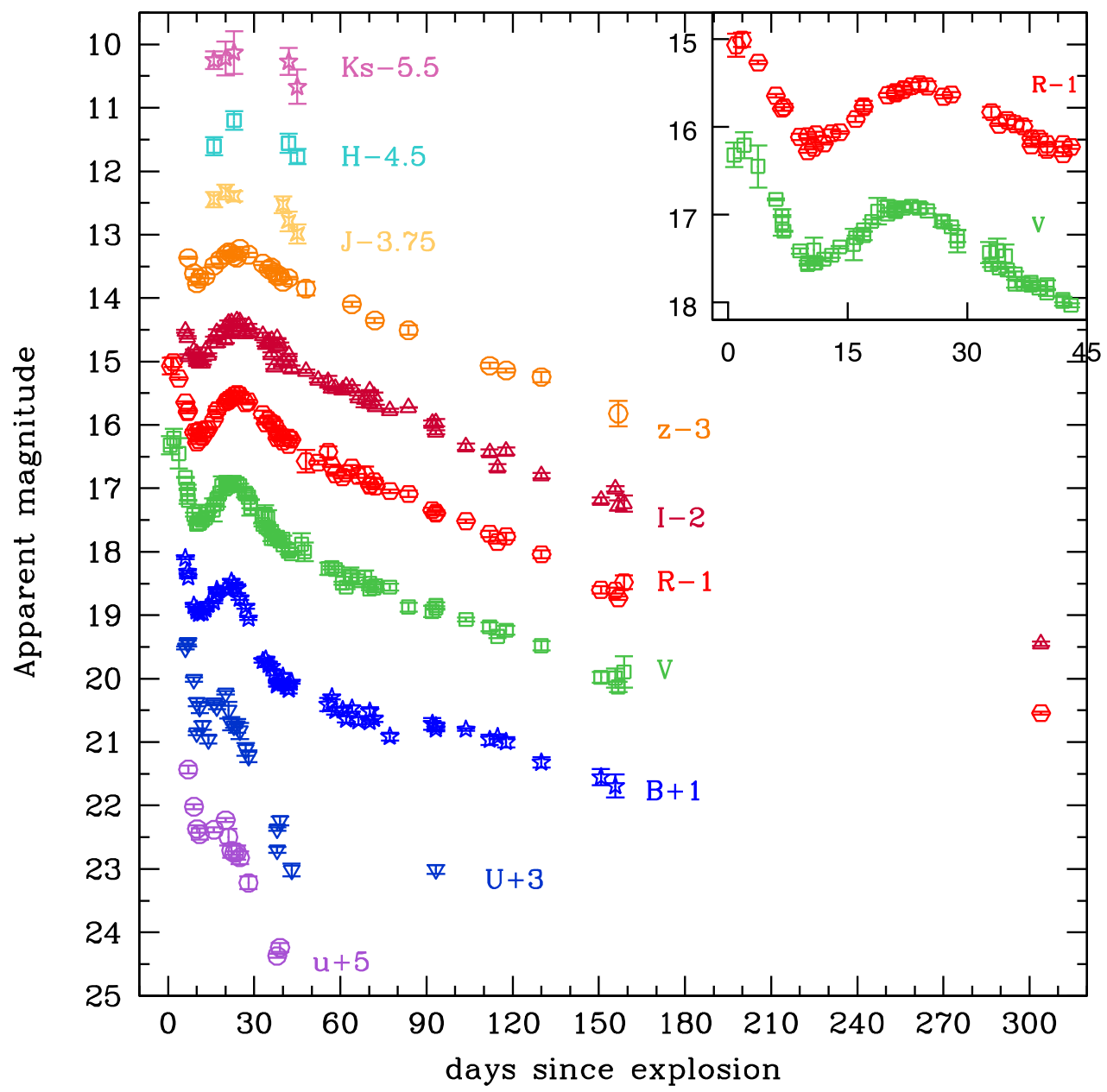

Figure 2. Optical-NIR light curves of SN 2011 fu. The assumed explosion epoch is JD $=2455824.5 \pm 0.7$. The LCs have been shifted for clarity by the values indicated in the figure. The inset in the top-right corner of the figure is a zoom of the $V$ and $R$ LCs up to $45 \mathrm{~d}$.

provided by amateur astronomers (see inset in the top right of the figure).

By making low-order polynomial fits to the optical LCs, we have estimated the phases and magnitudes at which the minima after first peak and secondary maxima take place. The results are presented in Table 1 . Note that we obtain different absolute magnitudes at secondary peak than Kumar et al. (2013) due to the fact that we have adopted different distance and reddening towards the host, however, our values agree within the uncertainties. We also fitted low-order polynomials to the first peaks in the $R$ and $V$ LCs. In the $V$ band, the first peak at 16.3 mag is reached $\sim 2.3 \mathrm{~d}$ after explosion while in the $R$ band the 16.0 mag first peak is obtained $\sim 2.8 \mathrm{~d}$ post-explosion.

We have also estimated the decline rates of the tails of the $B V R I z$ LCs (Table 1). These rates are quite similar to those estimated for SN 2013df by Morales-Garoffolo et al. (2014), and are steeper than expected from ${ }^{56} \mathrm{Co}$ decay. This is a common characteristic to stripped-envelope SNe (e.g. SN 2008ax; Taubenberger et al. 2011) and is possibly due to increasing transparency for $\gamma$ rays in their lower mass ejecta.

\subsubsection{Colour curves}

Fig. 3 shows the intrinsic colour evolution of SN $2011 \mathrm{fu}$ and for comparison the data for other type IIb SNe such as SN 1993J (Lewis et al. 1994; Barbon et al. 1995; Richmond et al. 1994; Matthews et al. 2002; assumed explosion date $\mathrm{JD}_{\exp }=2449074.0$ ), SN 2008ax (Pastorello et al. 2008; Taubenberger et al. 2011; Tsvetkov et al. 2009; assumed explosion date $\mathrm{JD}_{\exp }=2454528.8$ ), SN 2011dh (Ergon et al. 2015; assumed explosion date $\mathrm{JD}_{\exp }=2455713.0$ ), and SN 2013df (Morales-Garoffolo et al. 2014; assumed explosion date $\mathrm{JD}_{\exp }=2456450.0$ ). The adopted extinctions along the line of site of the $\mathrm{SNe}$ plus their distance are the same as those given in table 7 of Morales-Garoffolo et al. (2014). All colour indices of SN 2011 fu show a smooth rise from explosion up to $\sim 40 \mathrm{~d}$, that is $\sim 15 \mathrm{~d}$ after secondary peak, and afterwards they have a bluewards trend.

\subsection{Pseudo-bolometric LC}

To obtain the pseudo-bolometric optical-NIR LC, first we converted the apparent magnitudes of SN $2011 \mathrm{fu}$ (corrected for extinction) to effective fluxes. At the phases at which there were no data, the missing points were obtained by interpolation, or as in the cases of the $U$ and $u$ bands at more than $\sim 90 \mathrm{~d}$, and NIR prior and past secondary maximum, by extrapolation assuming a constant colour from the data at the nearest epoch. The fluxes were integrated over wavelength following a trapezoidal rule, and finally converted to luminosities taking into account the adopted distance to the SN.

In Fig. 4, we present the pseudo-bolometric NIR-optical LC of SN 2011fu as well as those of SNe 1993J, 2008ax, 2011dh, and 
Table 1. Optical and NIR magnitudes of the minimum and secondary maximum of SN 2011fu, the corresponding times at which they occurred, and tail decline rates in the BVRIz LCs.

\begin{tabular}{lcccccr}
\hline Band & $t_{\min }^{a}$ & Apparent magnitude at minimum & $t_{\max }^{a}$ & $\begin{array}{c}\text { Apparent magnitude at } \\
\text { secondary maximum } \\
(\mathrm{mag})\end{array}$ & $\begin{array}{c}\text { Absolute magnitude at } \\
\text { secondary maximum } \\
(\mathrm{mag})\end{array}$ & $\begin{array}{c}\text { Decline tail } \\
{[\mathrm{mag}(100 \mathrm{~d})}\end{array}$ \\
& $(\mathrm{d})$ & $(\mathrm{mag})$ & $18.8 \pm 0.8$ & $17.29 \pm 0.01$ & $-17.58 \pm 0.16$ & - \\
\hline$U$ & $13.6 \pm 1.1$ & $17.90 \pm 0.01$ & $21.8 \pm 1.6$ & $17.52 \pm 0.01$ & $-17.26 \pm 0.15$ & $1.25 \pm 0.07$ \\
$B$ & $11.8 \pm 1.0$ & $17.96 \pm 0.01$ & $22.9 \pm 0.7$ & $16.92 \pm 0.01$ & $-17.76 \pm 0.15$ & $1.78 \pm 0.04$ \\
$V$ & $10.6 \pm 0.7$ & $17.55 \pm 0.01$ & $24.2 \pm 1.2$ & $16.53 \pm 0.01$ & $-18.08 \pm 0.15$ & $2.04 \pm 0.04$ \\
$R$ & $11.1 \pm 2.1$ & $17.18 \pm 0.01$ & $24.1 \pm 1.2$ & $16.42 \pm 0.01$ & $-18.12 \pm 0.15$ & $1.97 \pm 0.05$ \\
$I$ & $11.4 \pm 1.5$ & $16.95 \pm 0.01$ & $24.1 \pm 1.0$ & $16.29 \pm 0.01$ & $-18.22 \pm 0.15$ & $1.82 \pm 0.04$ \\
$z$ & $10.5 \pm 0.7$ & $17.29 \pm 0.01$ & $24.5 \pm 2.6$ & $16.08 \pm 0.01$ & $-18.38 \pm 0.15$ & - \\
$J$ & - & - & $29.6 \pm 0.7$ & $15.62 \pm 0.01$ & $-18.80 \pm 0.15$ & - \\
$H$ & - & - & $29.2 \pm 4.5$ & $15.56 \pm 0.01$ & $-18.84 \pm 0.15$ & - \\
$K_{S}$ & - & - & & & & \\
\hline
\end{tabular}

Notes. ${ }^{a} t_{\min }$ and $t_{\max }$ are calculated with respect to our adopted explosion date JD $=2455824.5 \pm 0.7$. The errors in the NIR are large due to less photometric coverage of the maxima.

${ }^{b}$ Considering the interval starting $\sim 40 \mathrm{~d}$ after explosion to $\sim 160 \mathrm{~d}$.

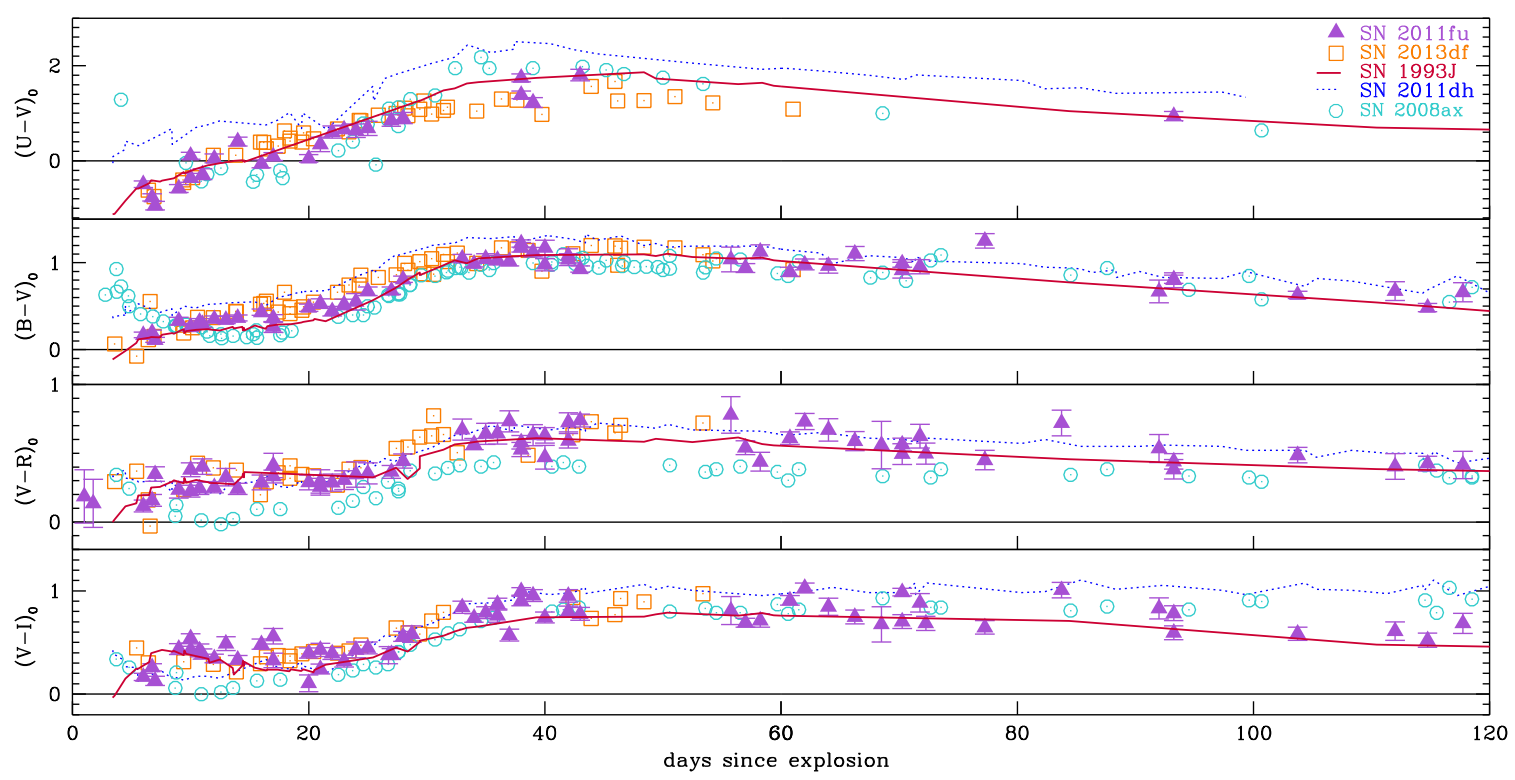

Figure 3. Comparison of the $(U-V)_{0},(B-V)_{0},(V-R)_{0}$, and $(V-I)_{0}$ colours of type IIb SNe 1993J, 2008ax, 2011dh, 2011fu, and 2013df. The colour of SN 2011 fu has been corrected for the assumed reddening $E(B-V)_{\text {Total }}=0.10_{-0.03}^{+0.04} \mathrm{mag}$, while the data and explosion epochs for the comparison supernovae were taken from the literature.

2013df, which we calculated in a similar way. In comparison to SNe 1993J and 2013df, SN 2011fu presents a longer cooling phase after the first peak, and a longer rise time to secondary peak than the rest of the objects, which makes SN 2011 fu the one with the highest ${ }^{56} \mathrm{Ni}$ mass synthesized in the explosion, in accordance with Kumar et al. (2013) and our modelling of the LC, which we present in Section 6.1. The slope of the LC tail starting at 40 and up to $\sim 300 \mathrm{~d}$ is a factor 1.1 steeper for SN 1993J, indicating as well that SN 2011fu ejected more mass in its explosion.

\section{SPECTROSCOPY}

\subsection{Spectral evolution of SN 2011 u}

In Fig. 5, we show the spectral evolution of SN 2011 fu ranging from $\sim 4$ to $\sim 304 \mathrm{~d}$ after explosion. The early spectra show a blue continuum and shallow features. Starting at around $6 \mathrm{~d} \mathrm{H} \alpha, \mathrm{H} \beta$ (with some possible Fe II contamination), $\mathrm{He}$ I $\lambda 5876$, Ca II $\mathrm{H} \& \mathrm{~K}$, Ca II NIR $\lambda \lambda \lambda 8498,8542,8662$, and Fe II $\lambda 5169$ appear to grow.
The $\mathrm{H} \alpha$ P-Cygni absorption component at $11.1 \mathrm{~d}$ shows a small trough at around $6300 \AA$ in addition to a broader and deeper one centred at approximately $6190 \AA$. These double $\mathrm{H} \alpha$ absorption features have been seen before in some type IIb SNe (e.g. SN 2011hs; Bufano et al. 2014) and have been claimed to be either due to Si II (Hachinger et al. 2012) or the presence of a double density distribution of hydrogen in the ejecta of the $\mathrm{SNe}$ as claimed for some type II $\mathrm{SNe}$ (e.g. Inserra et al. 2013). Note that in the first spectrum and the one at $14 \mathrm{~d}$, there is a narrow emission line at approximately the rest wavelength of $\mathrm{H} \alpha$. This line is also seen in some of our subsequent spectra, however, as we will explain below, we believe it is not associated with the SN or its circumstellar medium. After the LC's secondary maximum, at $34.1 \mathrm{~d}$, two new lines with fairly narrow absorptions at approximately 4840 and $4890 \AA$ are discernible, which we believe are associated with $\mathrm{Fe}$ II $\lambda 4924$ and $\mathrm{Fe}$ II $\lambda 5018+\mathrm{He}_{\mathrm{I}}$ $\lambda 5015$, respectively. In addition, there is an absorption that could be associated with $\mathrm{O}_{\mathrm{I}} \lambda 7774$. A major change occurs at $\sim 40 \mathrm{~d}$, when He I $\lambda 6678$ and He I $\lambda 7065$ appear and together with He I $\lambda 5876$ become progressively stronger. At $62 \mathrm{~d}$ a double-peaked emission 


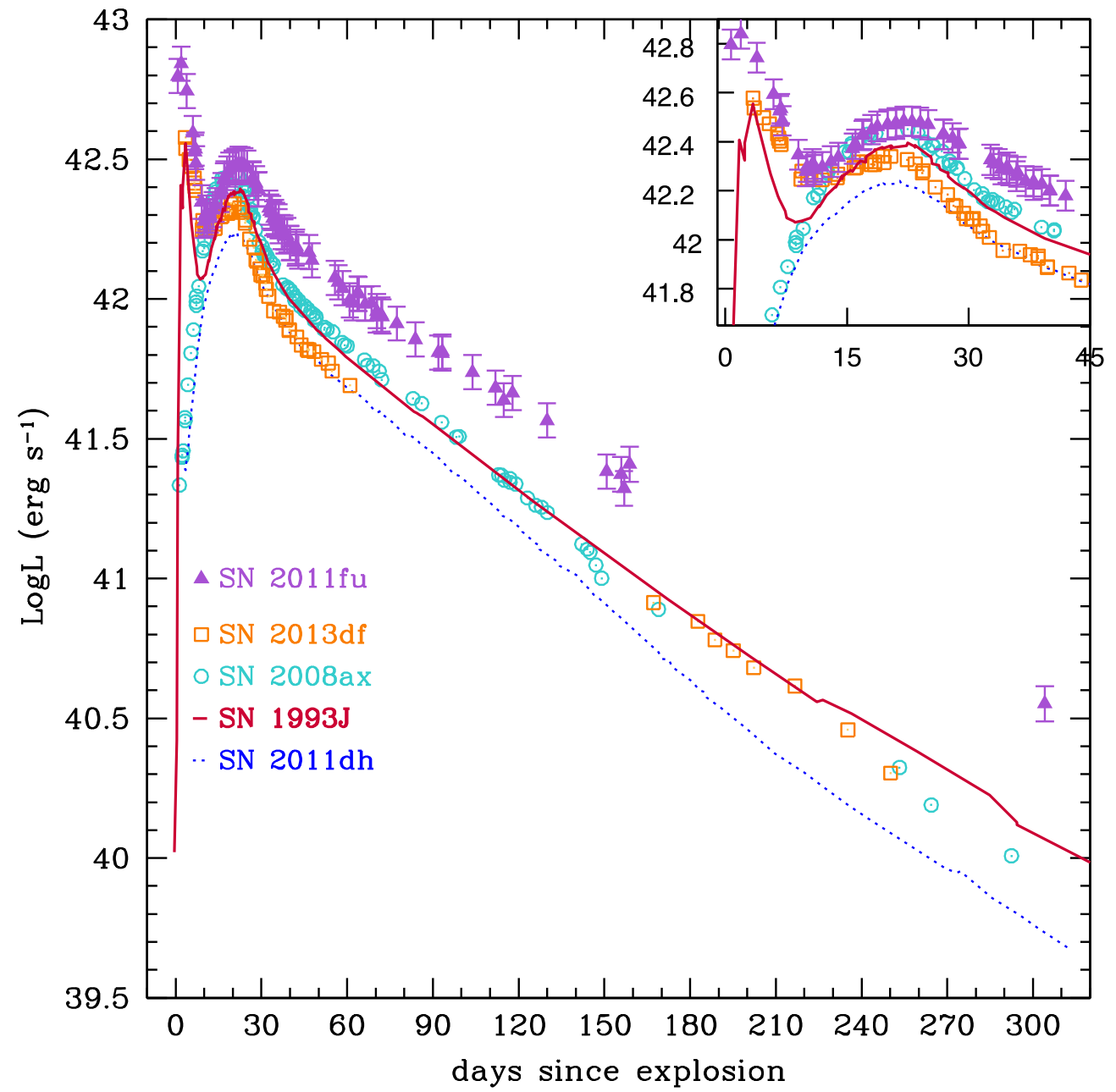

Figure 4. Pseudo-bolometric optical-NIR LC of SN $2011 \mathrm{fu}$ compared to type IIb SNe 2013df, 1993J, 2008ax, and 2011dh. The data and explosion epochs for the comparison supernovae were taken from the literature.

associated with [O I] $\lambda \lambda 6300,6364$ with components at approximately 6240 and $6300 \AA$ is observed, as well as an emission which has increased in intensity with respect to previous spectra, and is possibly associated with $[\mathrm{O}$ I] $\lambda 5577$. Starting at $103.8 \mathrm{~d}$, [N II] $\lambda \lambda 6548,6583$ produces an emission between $\sim 6400$ and $\sim 6700$ $\AA$ (Jerkstrand et al. 2015). At phase $155.8 \mathrm{~d}$, we notice a decrease in the intensity of $\mathrm{Na}$ I around $5890 \AA$ (which is possibly contaminated by residual $\mathrm{He}$ I $\lambda 5876$; Jerkstrand et al. 2015). In addition, we identify [Ca II] $\lambda \lambda 7291,7324$ and two strong emission lines, a narrow one at approximately the rest position of $\mathrm{H} \alpha$ (on top of $\mathrm{N}$ II $\lambda \lambda 6572,6583$ ) and the other at $\sim 6723 \AA$. In the last spectrum of our sequence, other than [O I] $\lambda \lambda 6300,6364,[\mathrm{Ca}$ II] $\lambda \lambda 7291,7324$, $\mathrm{O}_{\text {I }} \lambda 7774$, we also distinguish $\mathrm{Mg}$ I] $\lambda 4571,[\mathrm{Fe}$ II] $\lambda 7155$. The Ca II NIR line has diminished significantly from our previous spectra and is now blended with $\left[\mathrm{C}_{\mathrm{I}}\right] \lambda 8727$. The narrow emissions at $\mathrm{H} \alpha$ and the one at $\sim 6700 \AA$ are still present although the first seems to have diminished in intensity while the second has become broader and now is centred at approximately $6735 \AA$. The unresolved narrow $\mathrm{H} \alpha$ emission line seen in our two last spectra, and also observed in some of the earlier-time spectra, is detected mainly on nights in which the seeing was not good or the SN was faint, so it is probably due to contamination from a nearby $\mathrm{H}$ in region.

Concerning the line at $\sim 6700 \AA$, we note that in the nebular spectra of SN 2013df (Morales-Garoffolo et al. 2014) there was also an emission line at a similar wavelength, and it was interpreted as a redshifted component of the $\mathrm{SN} \mathrm{H} \alpha$ feature due to asymmetrical ejecta-circumstellar material interaction. However, since the line is detected in two different $\mathrm{SNe}$ it may be linked to a specific transition. In the models presented by Jerkstrand et al. (2015), there is an emission associated with $\left[\mathrm{S}_{\mathrm{II}}\right] \lambda \lambda 6716,6731$ about an order of magnitude weaker than the observed line. However, uncertainties in the temperature and ionization state might cause it to be stronger for SN 2011fu. Another possibility is due to contamination by the nearby $\mathrm{H}$ II region which we believe is causing the narrow $\mathrm{H} \alpha$ emission. In fact, in the $156 \mathrm{~d}$ spectrum both the narrow $\mathrm{H} \alpha$ and the $\sim 6700 \AA$ feature have similar FWHM and are unresolved suggesting a common origin not related to the $\mathrm{SN}$. Similar narrow $\mathrm{H} \alpha$ and $[\mathrm{S}$ II] $\lambda \lambda 6716,6731$ emissions caused by nearby $\mathrm{H}$ II regions are detected, e.g. in the spectra of the type IIb SNe 1987K (Filippenko et al. 1988) and 2008ax (Milisavljevic et al. 2010). However, in H II regions, $[\mathrm{S}$ II] $\lambda \lambda 6716,6731$ lines are less intense than $\mathrm{H} \alpha$ and this is not the case of the $304 \mathrm{~d}$ spectrum of SN 2011fu. In addition, the line at $\sim 6700 \AA$ at this phase is clearly resolved, for these reasons, an [ $\mathrm{S} \mathrm{II}_{\mathrm{II}} \lambda \lambda 6716,6731$ emission associated with the $\mathrm{SN}$ is the favoured possibility at $304 \mathrm{~d}$.

We have estimated the blackbody temperatures of the $\mathrm{SN}$ by fitting a blackbody function to the whole spectrum at each epoch. As can be seen in Fig. 6, the blackbody temperature evolves with 

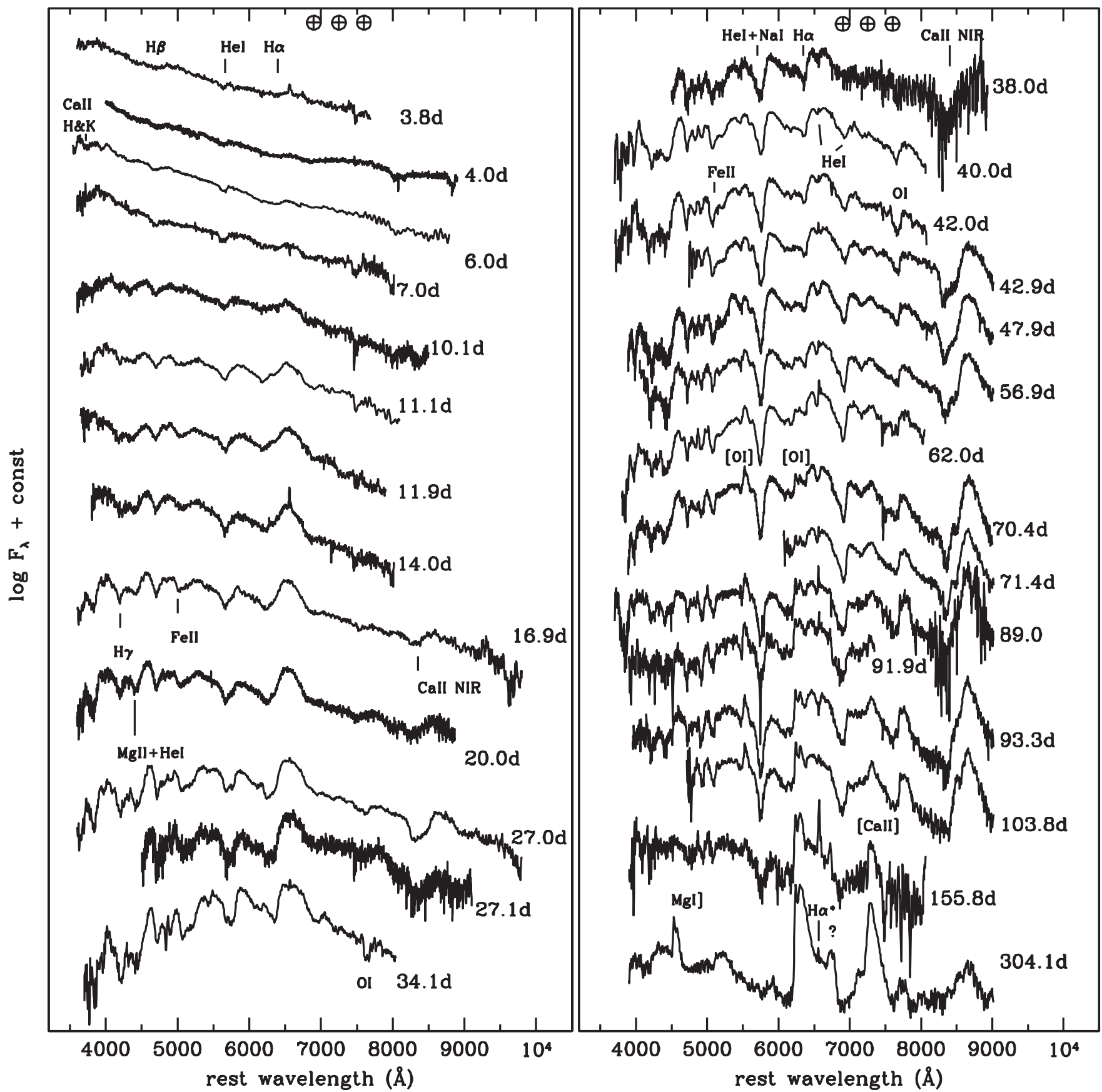

Figure 5. Optical spectral evolution of SN $2011 \mathrm{fu}$, where the most relevant features in the spectra are indicated. The wavelengths at which there are residual telluric features have been marked with $\oplus$. The spectra have been corrected for the host galaxy redshift. Epochs indicated in the plot are with respect to our assumed explosion date of JD $=2455824.5 \pm 0.7$. Spectra have been shifted vertically for clarity. $\mathrm{H} \alpha^{*}$ is the narrow $\mathrm{H} \alpha$ emission which we believe is associated to the $\mathrm{H}$ II region. The question mark at $\sim 6700 \AA$ marks the line we discussed in Section 5.1.

a steep decline up to approximately $40 \mathrm{~d}$ since explosion and after that, it roughly remains constant. The time evolution of the temperatures is in agreement with the colour evolution of the SN and with that derived by Kumar et al. (2013). As seen in Section 4.1.1, the colours redden during approximately the first $40 \mathrm{~d}$ and after that they are practically constant with a slight trend towards the blue. In the first $\sim 10 \mathrm{~d}$, the trend of the blackbody temperature is opposite to SN 2011dh (Ergon et al. 2014). Note that during this phase the LC of SN 2011fu is in the adiabatically powered declining phase to the minimum after the first peak while the pseudo-bolometric LC of SN 2011dh is radioactively powered.

\subsection{Velocities}

From the minima of the P-Cygni absorptions of $\mathrm{H} \alpha$ and $\mathrm{He}$ I $\lambda 5876$, we have an estimate of the velocities of the ejected material. Our results are presented in Fig. 7 together with the velocities for 


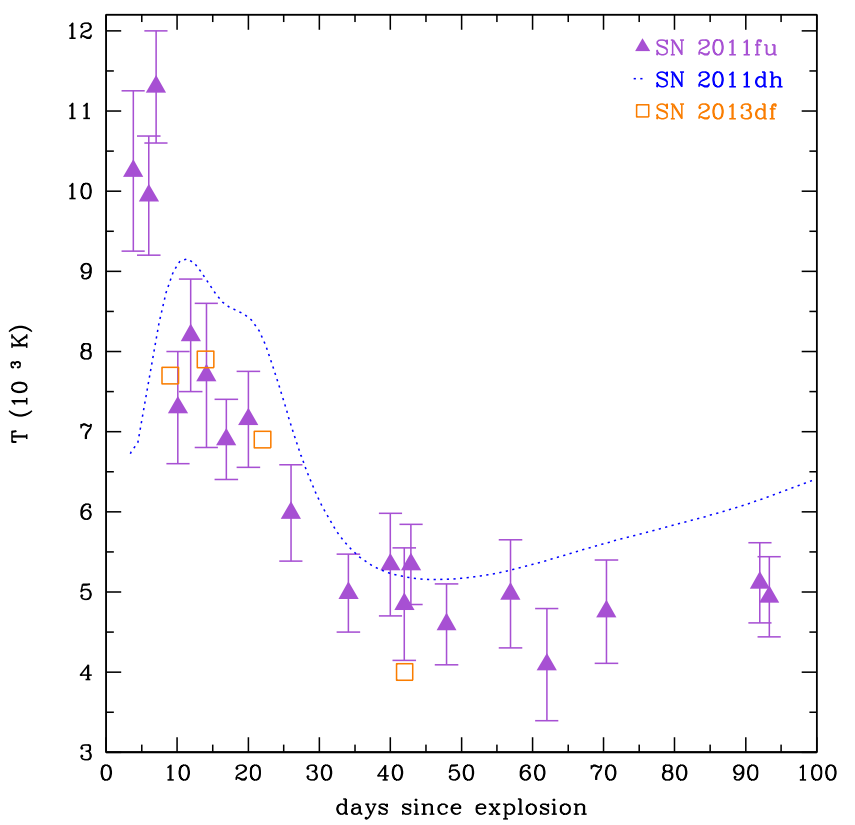

Figure 6. Blackbody temperature evolution of SN 2011fu with respect to our assumed explosion date, JD $=2455824.5 \pm 0.7$, compared to those of SNe 2011dh and 2013df.

SNe 1993J (Barbon et al. 1995; Taubenberger et al. 2011), 2008ax (Taubenberger et al. 2011), 2011dh (Ergon et al. 2014), and 2013df (Morales-Garoffolo et al. 2014). Note that the first two He I $\lambda 5876$ velocities reported in the figure were obtained from shallow, low contrast profiles, and in consequence are uncertain.

As we can see in Fig. 7, both the $\mathrm{H} \alpha$ and $\mathrm{He}$ I $\lambda 5876$ velocities we have derived are overall similar to those for the comparison SNe. Between 12 and $28 \mathrm{~d}$, we note that the $\mathrm{H} \alpha$ velocities for SN $2011 \mathrm{fu}$ are around $2000 \mathrm{~km} \mathrm{~s}^{-1}$ higher than those obtained with SYNOW by Kumar et al. (2013). From $\sim 40$ d on, the velocities remain constant and similar to those of SN 1993J but lower than the ones derived for SN 2011dh. We would like to point out that around a month past explosion the $\mathrm{He}$ I $\lambda 5876$ absorption component has a complex profile with a double trough, however, also in these cases the values reported in the figure are those obtained by adjusting a single Gaussian to the whole profile. Thus, the velocities measured for $\mathrm{He}$ I $\lambda 5876$ may have an added uncertainty due to the possible contamination by $\mathrm{Na}$ I $\lambda \lambda 5890,5896$, especially at later times. Noteworthy is the fact that for SN 2011dh, the hydrogen lines were never seen below $11000 \mathrm{~km} \mathrm{~s}^{-1}$, whereas the helium lines were always constrained to lower velocities. In fact, the modelling of the $\mathrm{SN}$ data suggested that $11000 \mathrm{~km} \mathrm{~s}^{-1}$ marked the interface between the H-rich envelope and the He core. For SN 2011fu, however, we cannot establish an analogous boundary based solely on the observed velocities, since in the velocity space $\mathrm{H} \alpha$ is not separated from He.

The photospheric velocities are expected to be similar to those derived from the $\mathrm{Fe}$ II $\lambda 5169$ line (see e.g. fig. 14 of Dessart \& Hillier 2005), these are shown in the right-hand panel of Fig. 7. As can be seen in this figure, the velocities of Fe II for SN 2011fu are overall comparable with those of our selected sample of type IIb SNe. We notice again that up to $30 \mathrm{~d}$ they are lower than the ones derived by Kumar et al. (2013) by about $2000 \mathrm{~km} \mathrm{~s}^{-1}$.

In summary, the expansion velocities of SN 2011fu are similar to those of other type IIb SNe.

\subsection{Comparison to other type IIb SNe}

In Fig. 8, we present a comparison of spectra of SN 2011fu at three different phases in its evolution along with those of other type IIb SNe downloaded from WISEREP ${ }^{5}$ (Yaron et al. 2012). As can be seen in the top panel of the figure, the spectra of SNe 1993J, 2011fu, and 2013df exhibit a blue almost featureless continuum with shallow hydrogen and helium lines (note that the spectrum of SN 2013df corresponds to a later phase). The coeval spectra of SNe 2008ax and 2011dh show much stronger lines and redder continuum, reflecting the absence of the first peak of the LC. In the middle panel of Fig. 8, we have depicted a spectral comparison at an intermediate phase $(\sim 40 \mathrm{~d})$. Although there is an overall resemblance in the spectra, the intensity of the lines varies from one $\mathrm{SN}$ to the other, indicating differences in the temperature of their ejecta. Specifically, SN 2011 fu has the weakest $\mathrm{H} \alpha$ and $\mathrm{He}$ I $\lambda 5876$ absorptions at this phase. Although not shown in the figure, we have also compared a spectrum of SN $2011 \mathrm{fu}$ at $22 \mathrm{~d}$ with the other type $\mathrm{IIb} \mathrm{SNe}$ at similar phases. Again, we have found the intensity of the $\mathrm{H} \alpha$ and $\mathrm{He}$ I $\lambda 5876$ lines to be lower than for the comparison SNe. For the nebular spectra (bottom panel of Fig. 8), in the case of SN $2011 \mathrm{fu}$, the [O I] $\lambda \lambda 6300,6364$ emission profile is double peaked, as it is for SN 2008ax. The Mg I] $\lambda 4571$ profile for SN 2011fu is also double peaked and again more similar to that of SN 2008ax. Interestingly and as noted above, SN 2011 fu shows an emission line at $\sim 6700 \AA$ similarly to SN $2013 \mathrm{df}$, although the line is broader in SN $2011 \mathrm{fu}$.

\subsection{Line profiles}

In the left-hand panel of Fig. 9 we present the evolution of SN 2011 fu's [O I] $\lambda \lambda 6300,6364$ nebular profile in velocity space. As seen in the figure, the profile shows two peaks, one at approximately $0 \mathrm{~km} \mathrm{~s}^{-1}$ and the other at $\sim-2800 \mathrm{~km} \mathrm{~s}^{-1}$. In the middle panel of Fig. 9, we show the nebular profiles of [O I] $\lambda 5577$ and $\mathrm{Mg}$ I] $\lambda 4571$. Finally, in the right-hand panel of the figure we have artificially added a component with $1 / 3$ the intensity of the original [O I] $\lambda 5577$ and $\left.\mathrm{Mg}_{\mathrm{I}}\right] \lambda 4571$ profiles and redshifted by $3000 \mathrm{~km} \mathrm{~s}^{-1}$, in order to compare them with the [O I] $\lambda \lambda 6300,6364$ doublet. As can be seen in this last panel, the shapes of the oxygen and magnesium profiles are quite similar to one another. Taubenberger et al. (2009) already found a great similarity between the oxygen and magnesium profiles in nebular spectra of some stripped-envelope SNe, supporting the idea that $\mathrm{Mg}$ and $\mathrm{O}$ have similar spatial distributions within their ejecta, which is expected from the models (Maeda et al. 2006). Moreover, in the case of the type IIb SN 2011dh the components of the small-scale fluctuations of the $\left[\mathrm{O}_{\mathrm{I}}\right] \lambda \lambda 6300,6364$ and $\left.\mathrm{Mg} \mathrm{I}\right]$ $\lambda 4571$ in its late-time spectra coincide, which in addition to the results of Jerkstrand et al. (2015) imply that the oxygen lines are mostly formed in the $\mathrm{O} / \mathrm{Ne} / \mathrm{Mg}$ zone.

One possibility to explain the shape of the profiles of SN $2011 \mathrm{fu}$ is that the bulk of oxygen and magnesium is distributed in spherically symmetric expanding ejecta but that there is a clump with emission from both these elements expanding at $\sim 2800 \mathrm{~km} \mathrm{~s}^{-1}$ towards the observer. A second possibility is that the radioactivity exciting the lines is distributed asymmetrically. Given the similarity of the [O I] $\lambda \lambda 6300,6364$ and the $\left[\mathrm{O}_{\mathrm{I}}\right] \lambda 5577$ and $\left.\mathrm{Mg} \mathrm{I}\right] \lambda 4571$ when an artificial component is added to the last two, an $\mathrm{H} \alpha$ high-velocity absorption, as found by Maurer er al. (2010) for some type IIb SNe, is not likely

\footnotetext{
${ }^{5} \mathrm{http}: / /$ wiserep.weizmann.ac.il/spectra/list
} 

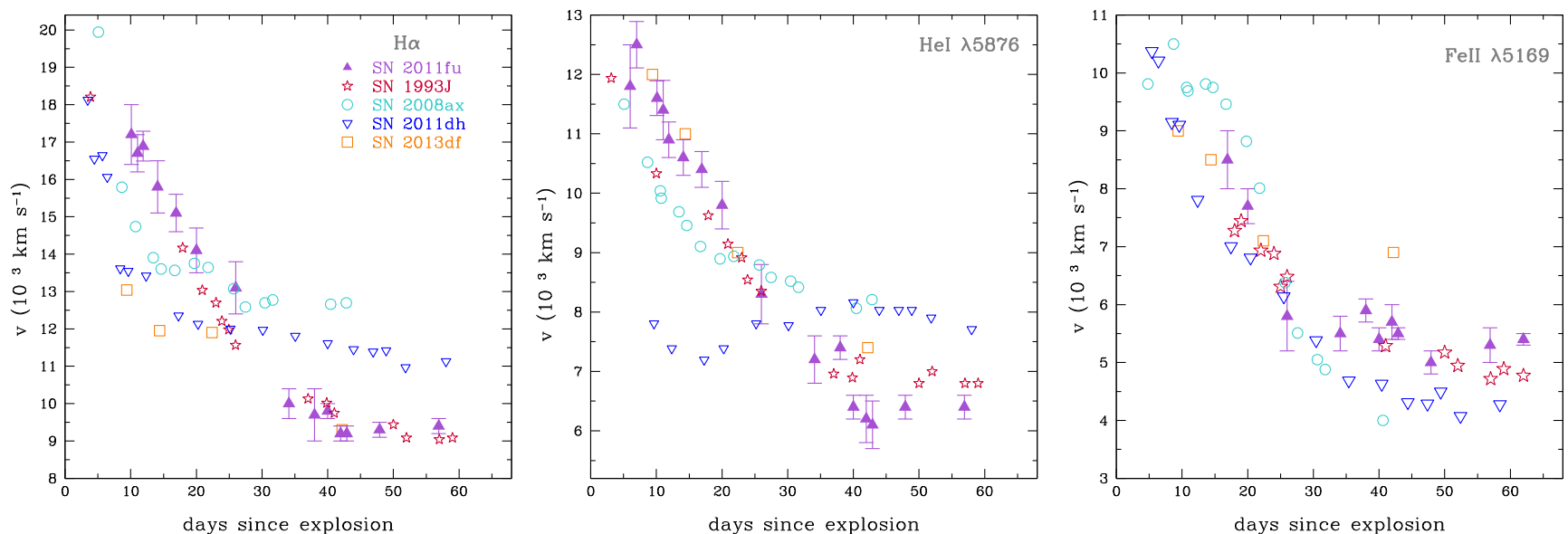

Figure 7. Velocity evolution of $\mathrm{H} \alpha$, He I $\lambda$ 5876, and Fe II $\lambda 5169$ for SN 2011 fu compared to those of SNe 1993J, 2008ax, 2011dh, and 2013df.

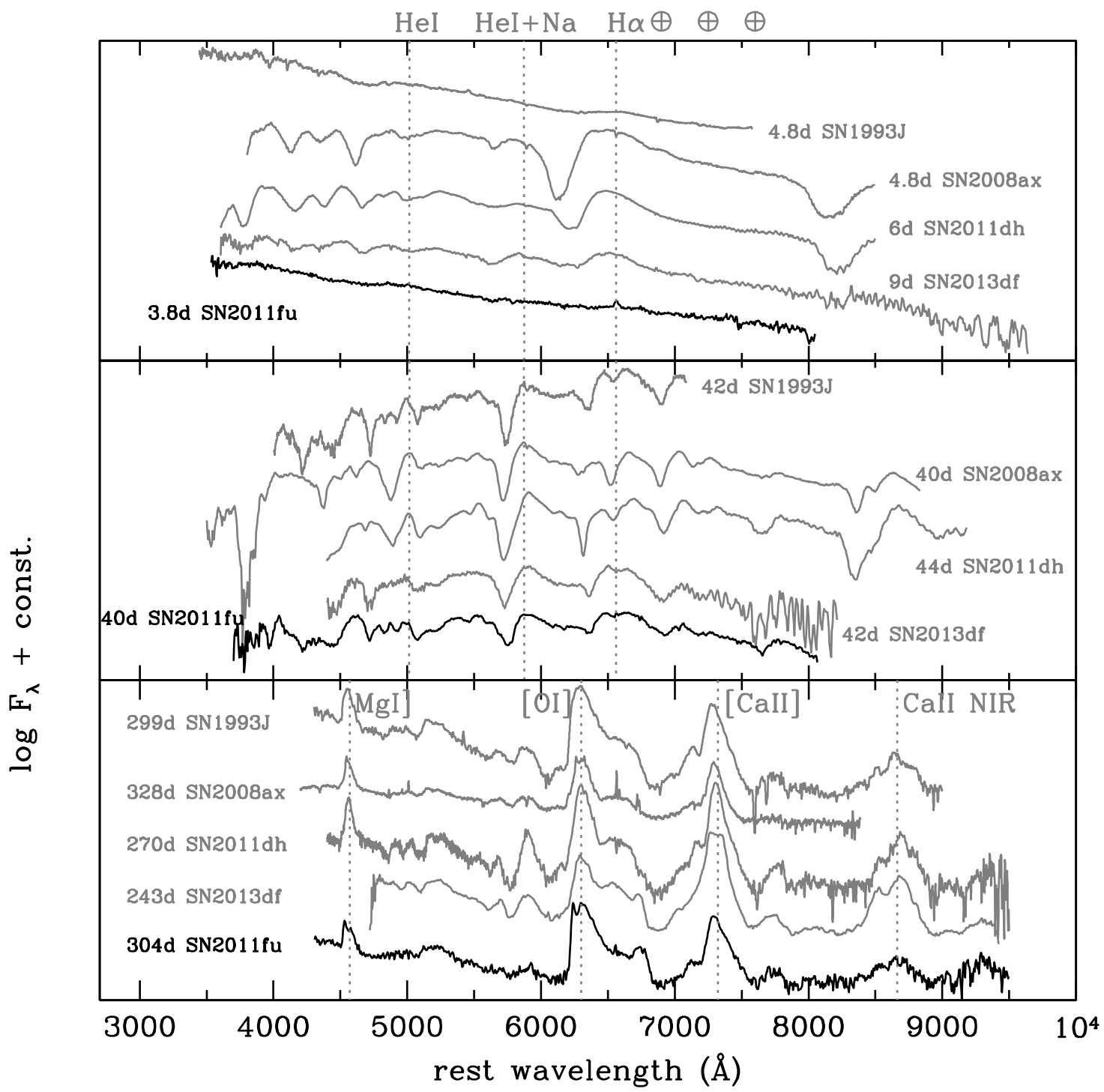

Figure 8. Comparison of early (5-9 d since explosion), intermediate (40-44 d after explosion), and late (299-398 d after explosion) spectra of SN 2011fu with those of type IIb SNe 1993J, 2008ax, 2011dh, and 2013df. The original references for the spectra of the comparison SNe are: Barbon et al. (1995) for SN 1993J at 4.8 and $42 \mathrm{~d}$, and an unpublished spectrum taken at the $1.82 \mathrm{~m}$ telescope in Asiago (Italy); Taubenberger et al. (2011) for SN 2008ax at 4.8 and 40 d, Modjaz et al. (2014) for SN 2008ax at 328 d; Ergon et al. (2014) for SN 2011dh at 6 and 44 d, Shivvers et al. (2013) for SN 2011dh at 270 d, and Morales-Garoffolo et al. (2014) for SN 2013df's spectra. The spectra have been dereddened, redshift corrected and shifted vertically for clarity. 


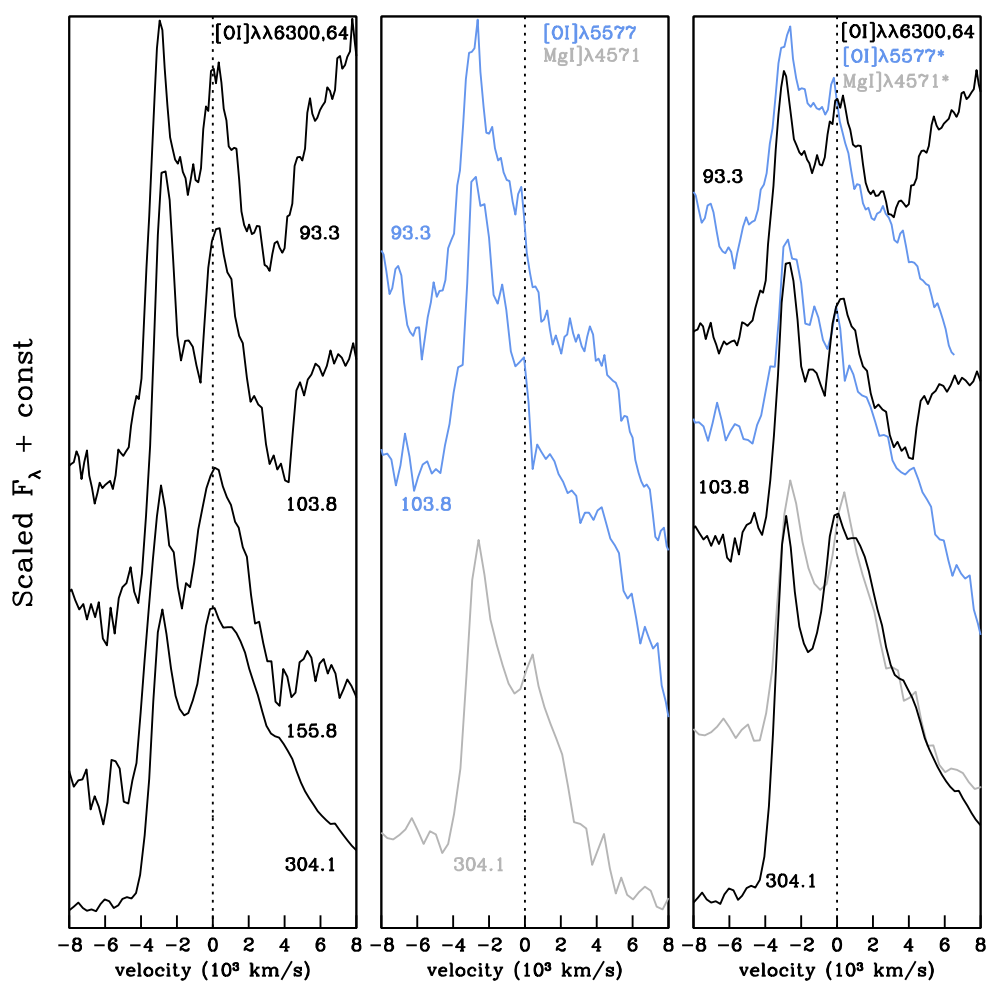

Figure 9. Left-hand panel: evolution of the late-time profiles of [O I] $\lambda \lambda 6300,6364$ in velocity space. The $0 \mathrm{~km} \mathrm{~s}^{-1}$ vertical line corresponds to $6300 \AA$. Middle panel: [O I] $\lambda 5577$ and $\mathrm{Mg}$ I] $\lambda 4571$ profiles between 93 and $304 \mathrm{~d}$ since explosion. Right-hand panel: comparison of the [O I] $\lambda \lambda 6300,6364$ profiles with [O I] $\lambda 5577^{*}$ and $\mathrm{Mg}$ I] $\lambda 4571^{*}$, which are the original profiles plus an artificial component, scaled to $1 / 3$ the intensity and redshifted by $3000 \mathrm{~km} \mathrm{~s}^{-1}$, added to account for the doublet nature of [O I] $\lambda \lambda 6300,6364$.

the principal cause of the double-peaked $\left[\mathrm{O}_{\mathrm{I}}\right] \lambda \lambda 6300,6364$ line, although it could be contributing to the profile. On the other hand, the narrow blueshifted component of the $\left[\mathrm{O}_{\mathrm{I}}\right] \lambda \lambda 6300$ carries a significant fraction of the flux even at $300 \mathrm{~d}$. Residual opacity in the core or line blocking explains blueshifted emission lines of stripped envelope and specifically type IIb SNe (Taubenberger et al. 2009; Jerkstrand et al. 2015). But in the case of SN 2011fu the persistence of the blueshifted emission from the [O I] $\lambda \lambda 6300,6364$ line over time suggests some degree of asymmetry in the SN ejecta. So, in principle, either clumping or an asymmetrical distribution of the radioactive material seems to be the most likely explanations for the profiles. Intriguingly, we have found two other $\mathrm{SNe}$ in the literature that have blueshifted double-peaked [O I] $\lambda \lambda 6300,6364$ profiles similar to SN 2011fu: types Ib SN 1996aq (Taubenberger et al. 2009) and SN 2005bf (Modjaz et al. 2008; Milisavljevic et al. 2010) at 216 d. For SN 1996aq, Taubenberger et al. (2009) speculated that the most likely explanation for its profile shape is that a clump is moving in the line of site at high velocity, and this is probably also the case for SNe $2011 \mathrm{fu}$ and 2005bf.

Finally, we note that the shape of SN 2011fu's [Ca II] $\lambda \lambda 7291$, 7324 line (not shown in the figure) is not similar to the oxygen and magnesium profiles, implying that, as expected, they are forming at different locations within the ejecta.

\section{DISCUSSION}

\subsection{Hydrodynamical modelling of the pseudo-bolometric LC}

One approach to derive physical parameters of $\mathrm{SNe}$ is to compare LCs and expansion velocities derived from hydrodynamical models with observations. From this comparison, it is possible to constrain the explosion energy, the ejecta, and the nickel masses as well as the distribution of the radioactive material. These parameters can be estimated by focussing the modelling around the 'main peak' of the LC (i.e. the nickel powered phase). Information on the size of the progenitor is given by the post-shock breakout cooling phase.

We calculated a set of hydrodynamical models using as initial structures those derived from stellar evolutionary calculations. A one-dimensional Lagrangian Local Thermodynamical Equilibrium (LTE) radiation hydrodynamics code (Bersten, Benvenuto \& Hamuy 2011) is used to explode the initial configuration. The code allows to calculate consistently the shock wave propagation in the stellar interior, the shock breakout, and the posterior phases of the LC evolution until the SN becomes nebular. An update of the microphysics (equation of state and opacities) appropriate for the study of stripped-envelope $\mathrm{SNe}$ was incorporated in the code by Bersten et al. (2012). As pre-SN structure we adopted helium (He) stars with a thin hydrogen envelope $\left(<1 \mathrm{M}_{\odot}\right)$ which have successfully reproduced the LCs and the spectral features of other SNe IIb (see e.g. Shigeyama et al. 1994; Blinnikov et al. 1998, and more recently Bersten et al. 2012). The He core models used here were calculated by Nomoto \& Hashimoto (1988) following the stellar evolution until the collapse of the core. We have smoothly attached a low-mass hydrogen-rich envelope in hydrostatic and thermal equilibrium to the He core to take into account the thin $\mathrm{H}$-envelope required for a SN IIb.

First we focus our analysis on the modelling of the LC and the photospheric velocities around the secondary peak without taking into account the cooling part of the LC. Fig. 10 shows the results of the LC modelling for three different He core masses, 4 (He4), 5 

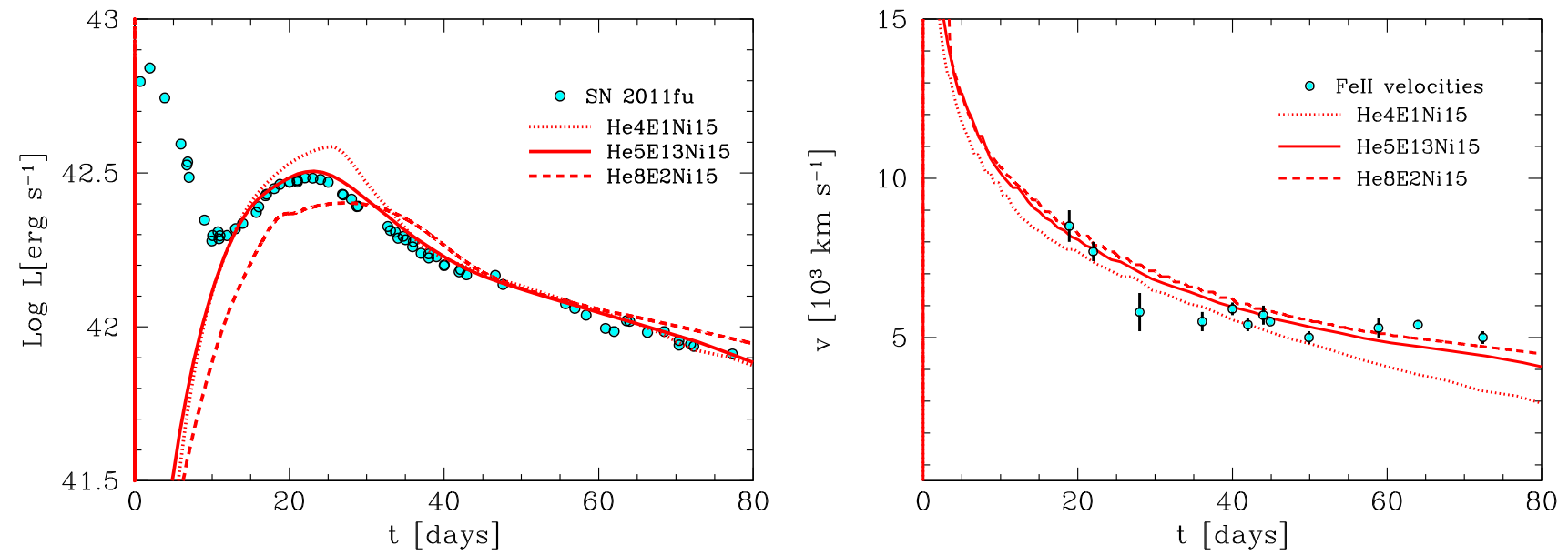

Figure 10. Left-hand panel: observed bolometric light curve of SN $2011 \mathrm{fu}$ (dots) compared with the results of the light-curve calculations for models He4E1Ni15 (dotted line), He5E13Ni15 (solid line), and He8E2Ni15 (dashed line) omitting the first peak. Right-hand panel: evolution of the photospheric velocity for models He4E1Ni15 (dotted line), He5E13Ni15 (solid line), and He8E2Ni15 (dashed line) compared with measured Fe II line velocities of SN $2011 \mathrm{fu}$ (dots).

(He5), and $8 \mathrm{M}_{\odot}(\mathrm{He} 8)$, which correspond to the stellar evolution of single stars with main-sequence masses of 15,18 , and $25 \mathrm{M}_{\odot}$, respectively. For each model, different explosion energies and ${ }^{56} \mathrm{Ni}$ masses were explored. Here, we show the best set of parameters for each of the models. Specifically, an explosion energy of 1, 1.3, and 2 foe $\left(1\right.$ foe $\left.=1 \times 10^{51} \mathrm{erg}\right)$ and a ${ }^{56} \mathrm{Ni}$ mass of $0.15 \mathrm{M}_{\odot}{ }^{6}$ were found for model He4 (He4E1Ni15), He5 (He5E13Ni15), and He8 (He8E2Ni15), respectively. As can be seen in the figure, model $\mathrm{He} 5$ provides the best representation of the observed data. The $\mathrm{He} 8$ model is too massive to reproduce the LC unless we assume a more energetic explosion but this would fail to fit the expansion velocities. On the other hand, a lower mass model, $\mathrm{He} 4$, gives a worse fit of the LC and underestimates the early photospheric velocities.

Our favoured model, $\mathrm{He} 5$, is an $\mathrm{H}$-free object with an initial radius of $\approx 2 R_{\odot}$. Although, this model gives a good representation of the data around the secondary peak, it fails to reproduce the cooling phase due to the compact structure of the progenitor. Fig. 11 shows the fits to the LC for models with different progenitor radius. The size of the progenitor was modified by attaching thin $\mathrm{H}$-rich envelopes to the core of the He5 model (solid line). Note that the presence of a thin envelope mostly affects the shape of the LC during the cooling phase. Fig. 11 shows models with radii of 400,450 , and $500 \mathrm{R}_{\odot}$ and envelope masses $\left(M_{\text {env }}\right)$ of $\approx 0.3 \mathrm{M}_{\odot}$. The model with $R=450 R_{\odot}$ provides the closest match to the data. However, all the models give a initial peak brighter than the observations. The differences may be due to uncertainties in the bolometric calculation (note that we have no UV data for the $\mathrm{SN}$, which are important at these early phases) or/and limitations of the model such as the LTE approximation or variation of the density profile of the outermost layers, among other. It is noteworthy that to reproduce the early phase of the LC it was not only needed to assume an extended envelope but it was also necessary to adopt a slightly more massive envelope than in previous modelling of $\mathrm{SNe}$ IIb, where, e.g. $M_{\text {env }}=0.1 \mathrm{M}_{\odot}$ was required for SN 2011dh and SN 1993J (Bersten et al. 2012; see also Nakar \& Piro 2014). The need for a more massive envelope was mainly due to the high luminosity of the minimum after primary maximum of the LC. For

\footnotetext{
${ }^{6}$ We have estimated the uncertainty of the ${ }^{56} \mathrm{Ni}$ mass by considering only
} the photometric errors to be $0.02 \mathrm{M}_{\odot}$.

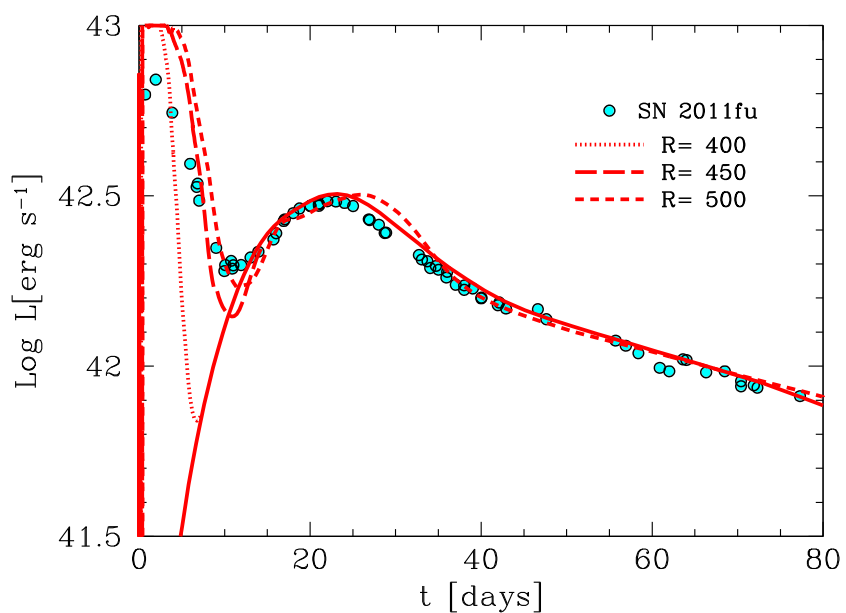

Figure 11. Observed bolometric LC of SN 2011 fu (dots) compared with the bolometric LCs for models with the same physical parameters than our preferred model (He5E13Ni15; solid line), but different initial radii. The radius variation is accomplished by attaching thin $\mathrm{H}$-rich envelopes to the He5 model. An extended progenitor with $R \approx 450 \mathrm{R}_{\odot}$ (long dashed) is needed to reproduce the cooling phase of SN $2011 \mathrm{fu}$.

lower $M_{\text {env }}$, the cooling occurs faster and the minimum occurs at a lower luminosity even for large radius.

A summary of all the parameters obtained from the best core and envelope model that fit the observed LC is shown in Table 2. Similar to Kumar et al. (2013), we have obtained a kinetic energy which is relatively higher than that obtained for other type IIb SNe but still lower than those derived for SN 2011ei (Milisavljevic et al. 2013) and the type IIb hypernova SN 2003bg (Hamuy et al. 2009). The comparison to the pseudo-bolometric LCs of other type IIb $\mathrm{SNe}$ in Section 4.2 indicates that SN 2011 fu synthesized more ${ }^{56} \mathrm{Ni}$ in its explosion than SNe 1993J, 2008ax, 2011dh, and 2013df (cf table 7 of Morales-Garoffolo et al. 2014). The modelling of the LC corroborates this result. Note that we obtain a lower ${ }^{56} \mathrm{Ni}$ mass than Kumar et al. $\left(2013,0.21 \mathrm{M}_{\odot}\right)$ possibly due to the different distance and extinction to SN 2011fu that we have adopted while our estimate of the ejected mass is larger than theirs $\left(1.1 \mathrm{M}_{\odot}\right)$. 
Table 2. Explosion parameters and progenitor properties for the best-fitting models to the observed data of SN 2011fu.

\begin{tabular}{lc}
\hline Parameters & Explosion/Progenitor \\
\hline$E_{\text {kin }}\left(10^{51} \mathrm{erg}\right)$ & 1.3 \\
${ }^{56} \mathrm{Ni} \mathrm{mass}\left(\mathrm{M}_{\odot}\right)$ & 0.15 \\
$M_{\mathrm{ej}}\left(\mathrm{M}_{\odot}\right)$ & $3.5^{a}$ \\
Progenitor radius $\left(\mathrm{R}_{\odot}\right)$ & 450 \\
Initial mass $\left(\mathrm{M}_{\odot}\right)$ & 18 \\
Hydrogen envelope mass $\left(\mathrm{M}_{\odot}\right)$ & 0.3 \\
\hline
\end{tabular}

Note. ${ }^{a}$ Assuming that $1.5 \mathrm{M}_{\odot}$ collapsed to form a compact remnant and the rest of the mass that formed the core was ejected.

Concerning the progenitor radius, core mass, and hydrogen envelope, we have obtained overall larger values than those obtained by Kumar et al. (2013) with analytical models. Specifically, they obtained a progenitor radius of $\sim 150 \mathrm{R}_{\odot}$, an He core mass of $1 \mathrm{M}_{\odot}$, and a hydrogen envelope mass of $0.1 \mathrm{M}_{\odot}$. But as they noted, their results should be considered only order of magnitude estimates. With hydrodynamical modelling, we obtained a radius that is three times larger and consistent with that of an extended supergiant similarly to the progenitors of SNe $1993 \mathrm{~J}\left(\sim 600 \mathrm{R}_{\odot}\right.$; Maund et al. 2004; Van Dyk et al. 2013) and 2013df ( $550 \mathrm{R}_{\odot}$; Van Dyk et al. 2014). All in all, our calculations show that the progenitor of SN 2011fu was not a WR star but a supergiant.

\subsection{Comparison with late-time spectral models}

With the objective of better understanding the nature of the progenitor of SN $2011 \mathrm{fu}$ and its ejecta, we have compared the last three spectra of our sequence $(104,156,304 \mathrm{~d})$ with the late-time spectral models presented in Jerkstrand et al. (2015).

To begin with we compared our spectra with the three models with the same (best fit to SN 2011dh) settings for mixing, clumping, molecule, and dust, and differing only in progenitor mass $(12,13$, and $17 \mathrm{M}_{\odot}$ ). These are models $12 \mathrm{C}, 13 \mathrm{G}$, and 17A (see table 4 in Jerkstrand et al. 2015). In order to do this, we scaled the models accounting for differences in ${ }^{56} \mathrm{Ni}$ mass synthesized in the explosion, phase (if the difference in phase between models and spectra is $\Delta t$, the factor $\exp (-2 \Delta \mathrm{t} / 111.4)$ is used to scale the models $)$, and distances assumed for the models and the SN. The models have been calculated at a distance of $7.8 \mathrm{Mpc}$ and assuming an ${ }^{56} \mathrm{Ni}$ mass of $0.075 \mathrm{M}_{\odot}$ (since they were constructed to compare with SN 2011dh), while in Section 6.1 we have estimated $0.15 \pm 0.02 \mathrm{M}_{\odot}$ of ${ }^{56} \mathrm{Ni}$ from SN 2011fu. Overall, the model spectra are dimmer than the SN spectra. We note that for ${ }^{56} \mathrm{Ni}$ on the higher end of its uncertainty the flux difference between modelled and observed spectra diminishes. Given that the errors in the extinction are not large, it is likely not the cause of the discrepancy between the flux levels. Another possibility is that the ejecta structure of SN $2011 \mathrm{fu}$ is different than the one used in the models and the IIb SNe fit with the models. Note that the Jerkstrand et al. (2015) models have an imposed dust extinction of $\tau=0.25$ from $200 \mathrm{~d}$, if no dust is produced in SN $2011 \mathrm{fu}$ this introduces a 25 percent flux error for the last spectrum.

At all phases, the overall best-fitting model is $13 \mathrm{G}$. The oxygen lines produced by the $17 \mathrm{~A}$ model are more intense than those observed, while the $12 \mathrm{C}$ model underestimates the oxygen intensities. In Fig. 12, we present the three late-time SN spectra compared to the $13 \mathrm{G}$ spectral models at coeval phase. We have also indicated in the plot some of the most important features that arise in the late spectral modelling. ${ }^{7}$ In the middle panel of the figure, we have also plotted model 17A and $12 \mathrm{C}$ to reflect the discrepancy between the oxygen lines of the model and those observed of the spectrum. In Fig. 13, we present a comparison of the [O I] $\lambda \lambda 6300$, 6364 late-time luminosities of SN 20111fu and those corresponding to models $12 \mathrm{C}, 13 \mathrm{G}$, and $17 \mathrm{~A}$. All in all, these comparisons indicate that the progenitor of SN 2011fu was likely at the lower end of the tested range, with $M_{\text {ZAMS }} \sim 13 \mathrm{M}_{\odot}$. The model that best fits the observed LC, however, corresponds to a progenitor star with a $5 \mathrm{M}_{\odot}$ He core, i.e. $M_{\text {ZAMS }}=18 \mathrm{M}_{\odot}$. This value is higher but not inconsistent with the estimate we have obtained here. In fact, it is not surprising to have found different values for the progenitor's initial mass since the initial conditions and the methodology used in both modelling methods are rather different. The hydrodynamical modelling of the LC uses stellar evolutionary models with different progenitor $M_{\text {ZAMS }}$ and explodes these structures for different values of explosion energies and nickel masses to compare with the data of SN 2011fu. The greatest degeneracy in the model is between mass and energy, this is the reason for modelling both the LC and the expansion velocities. Another source of uncertainty is introduced by the initial stellar evolutionary models. The code treats in a very approximate way the radiation transportation but correctly simulates the explosion dynamics.

The nebular spectral models use ejecta with nucleosynthesis from the evolution and explosion of stars of different $M_{\text {ZAMs }}$. While the dynamic structure of the core is manually arranged to match the observed metal line widths in type IIb SNe $\left(\sim 3500 \mathrm{~km} \mathrm{~s}^{-1}\right)$ as well as to capture effects of macroscopic mixing and clumping seen in 2D simulations and inferred from observed line profiles, the envelope follows a profile obtained in a $1 \mathrm{D}$ explosion simulation by Bersten et al. (2012). Uncertainty is introduced by two sources: the dynamic structure of the ejecta and the margin of error in the spectral modelling. Since the model reproduces well the metal line profiles of SN $2011 \mathrm{fu}$, the core velocity of $3500 \mathrm{~km} \mathrm{~s}^{-1}$ is likely accurate. The envelope absorbs quite little of the gamma-ray energy (see appendix A in Jerkstrand et al. 2015) so an uncertainty in its density profile introduces only a moderate dispersion for the flux levels. Thus, the model uncertainty is likely dominated by the error in the ${ }^{56} \mathrm{Ni}$ mixing as well as molecule and dust formation. The ${ }^{56} \mathrm{Ni}$ mixing used in $13 \mathrm{G}$ is chosen to match both the diffusion phase LC and the nebular spectra of SN 2011dh, but may be somewhat different in SN $2011 \mathrm{fu}$.

It would be interesting to analyse whether the differences between the two modelling approaches persist if the post-explosion density structure of our preferred LC model for SN 2011fu is used as initial condition of the spectral modelling. However, this analysis is beyond the scope of this work. In any case, the mass estimates obtained from the two methods are consistent in the sense that we can discard the possibility of the progenitor being a single WR star (with $M_{\text {ZAMS }} \geq 25$ ).

To check for evidence of molecule formation in the ejecta of the SN, we compared models 12C (no molecular cooling; see table 4 of Jerkstrand et al. 2015) and 12D (with molecular cooling) with our late-time spectra. In the bottom panel of Fig. 12, the comparison at $300 \mathrm{~d}$ is shown. The oxygen lines are the ones that are most sensitive to molecule formation at 100 and $150 \mathrm{~d}$ along with [C $\mathrm{I}]$ $\lambda 8727$ at $300 \mathrm{~d}$. In our case, the model without molecular cooling

\footnotetext{
${ }^{7} \mathrm{H} \alpha *$ is the narrow $\mathrm{H} \alpha$ emission which we believe is associated with an $\mathrm{H}$ II region. The question mark at $\sim 6700$ marks the line we discussed in Section 5.1.
} 


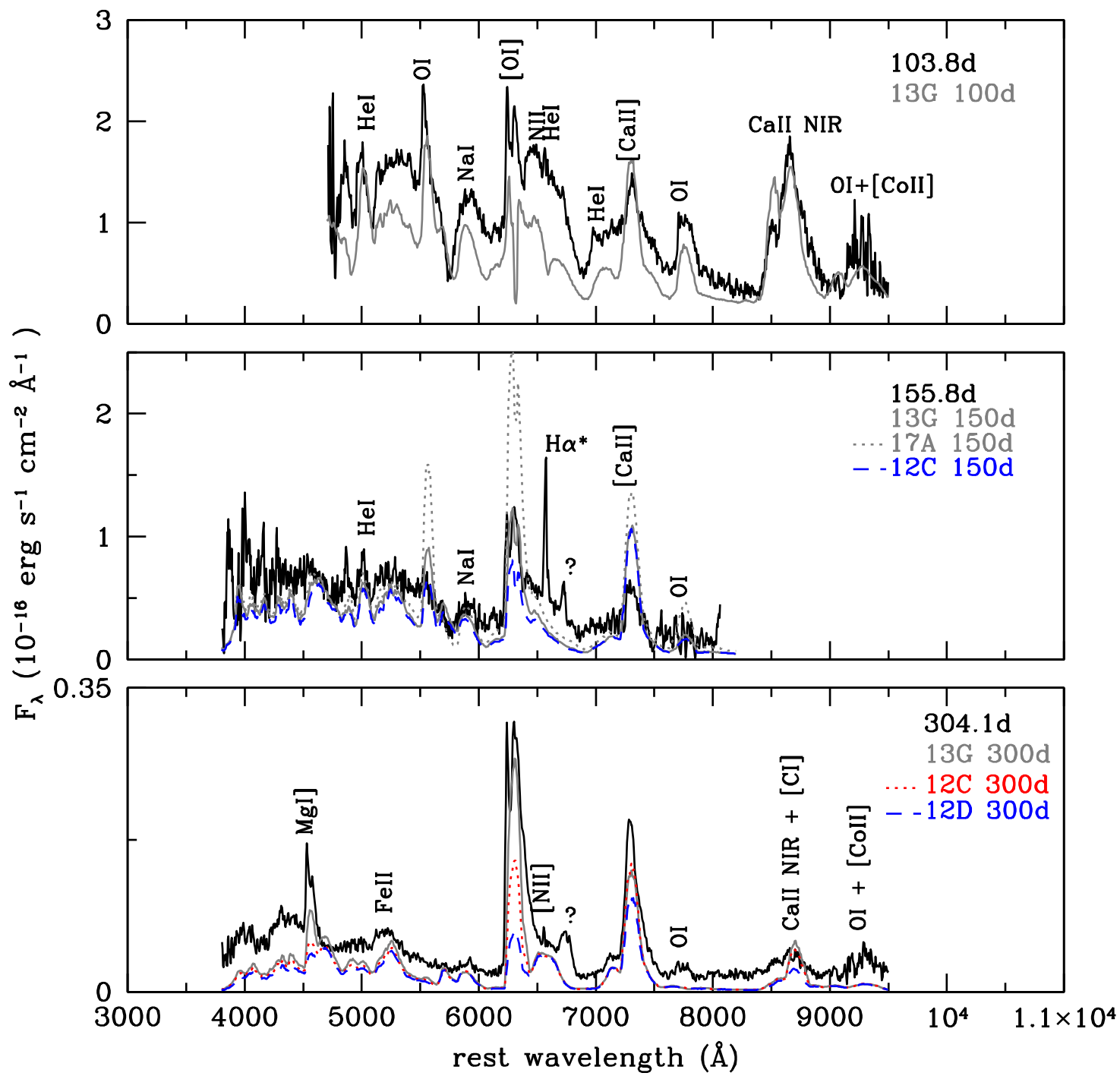

Figure 12. Late-time spectra of SN 2011 fu compared to the best-fitting spectral model 13G presented in Jerkstrand et al. 2015. In the middle panel we show our spectrum at $\sim 156 \mathrm{~d}$ and the three models that vary only in progenitor mass. In the bottom panel other, than model $13 \mathrm{G}$ we show models $12 \mathrm{C}$ and $12 \mathrm{D}$, which are the ones used to study molecule formation in the SN ejecta. The spectral models have been scaled accounting for difference in phase with respect to the SN spectra $(\Delta t)$ by the factor $\exp (-2 \Delta \mathrm{t} / 111.4)$, difference in ${ }^{56} \mathrm{Ni}$ synthesized mass, and distance assumed for the models and the SN.

is favoured, similarly to the case of SN $2011 \mathrm{dh}$. We also looked at the contrast factor or oxygen zone density, i.e. the density ratio between the metal zones in the core and the $\mathrm{Fe} / \mathrm{Co} / \mathrm{He}$ zone, but the models with different oxygen zone densities do not show palpable differences. However, we do note that the Mg I] $\lambda 4571$ line at $304 \mathrm{~d}$ is quite strong, which favours a high oxygen zone density, since the line is quite sensitive to this parameter, and this is in agreement with the results found for SN 2011dh in Jerkstrand et al. (2015).

In summary, the comparisons of our nebular spectra with the models presented in Jerkstrand et al. (2015) point to a progenitor with an initial mass of $\sim 13 \mathrm{M}_{\odot}$, likely no molecules were formed in the ejecta, and the oxygen zone density was possibly high.

\section{CONCLUSIONS}

In this paper, we have analysed optical and NIR data for the doublepeaked type IIb SN 2011 fu spanning from a few days to approximately $300 \mathrm{~d}$ after explosion. The double-peaked LC is brighter than those of other type IIb SNe such as SNe 1993J, 2008ax, 2011dh, and $2013 \mathrm{df}$. It also presents a longer cooling phase after primary peak than SNe 1993J and 2013df.

SN 2011fu's spectra evolve in a similar fashion to those of SN 1993J. During the first phases after explosion Balmer, $\mathrm{He}, \mathrm{Ca}$, and Fe lines are present in the spectra. At around $40 \mathrm{~d}$, He lines dominate the spectra although $\mathrm{H} \alpha$ is still present and persists up to later phases. Forbidden oxygen lines are clearly visible at $\sim 62 \mathrm{~d}$, which indicates that the $\mathrm{SN}$ is entering the nebular phase. We derived $\mathrm{H} \alpha$, $\mathrm{He}$ I $\lambda 5876$, and $\mathrm{Fe}$ II $\lambda 5169$ velocities from the absorption minima of the P-Cygni profiles in SN 2011 fu's spectra, which are consistent with the results obtained for other type IIb SNe. At late times, the most important features are [O I] $\lambda \lambda 6300,6364$, and [Ca II] $\lambda \lambda 7291$, 7324. In addition in our latest spectrum taken at $304 \mathrm{~d}, \mathrm{Mg}$ I] $\lambda 4571$ and a line that could be due to $[\mathrm{S}$ II] $\lambda \lambda 6713,6730$ are detected.

The nebular profiles of oxygen and magnesium indicate that the oxygen/magnesium zone is clumped. Calcium lines do not show similar profiles indicating, as expected, that they are formed at a different location. 


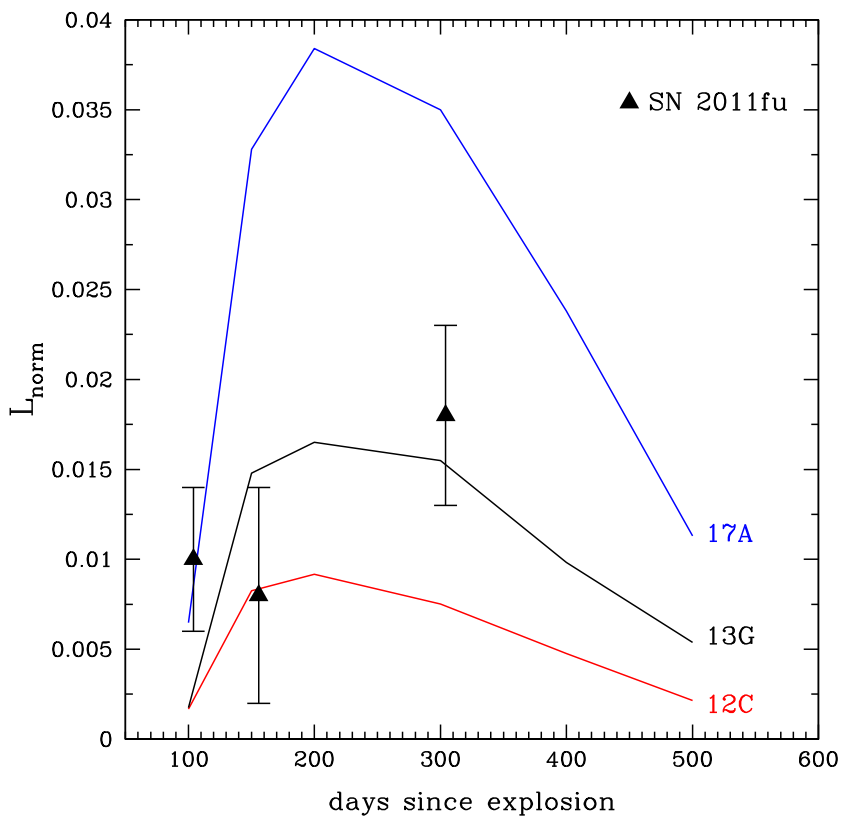

Figure 13. Late-time [O I] $\lambda \lambda 6300,6364$ luminosities of SN 2011fu (triangles) compared to model tracks $12 \mathrm{C}, 13 \mathrm{G}$, and $17 \mathrm{~A}$ of Jerkstrand et al. (2015).

Thanks to the hydrodynamical modelling of the pseudobolometric LC we conclude that SN 2011fu was the explosion of an extended object $\left(R \approx 450 \mathrm{R}_{\odot}\right)$, with an He core mass of $5 \mathrm{M}_{\odot}$ $\left(M_{\text {ZAMS }} \approx 18 \mathrm{M}_{\odot}\right)$ and an ejecta mass of $3.5 \mathrm{M}_{\odot}$, an explosion energy of $1.3 \times 10^{51} \mathrm{erg}$, and an ${ }^{56} \mathrm{Ni}$ mass of $0.15 \mathrm{M}_{\odot}$. In comparison with other $\mathrm{SNe}$ IIb, this object seems to be a bit more massive, more energetic and to have produced more ${ }^{56} \mathrm{Ni}$. The stellar envelope of its progenitor also seems to have been more massive than for other type IIb SNe $\left(\sim 0.3 \mathrm{M}_{\odot}\right)$, implying the presence of more $\mathrm{H}$ in the $\mathrm{SN}$ ejecta.

We have compared our late-time spectra $(\geq 100 \mathrm{~d})$ with latetime spectral models for type IIb SNe presented in Jerkstrand et al. (2015). From this analysis, the overall best-fitting value of the progenitor mass is $13 \mathrm{M}_{\odot}$, similar to the $M_{\text {ZAMS }}$ values that have previously been derived for SNe 1993J, 2008ax, and 2011dh

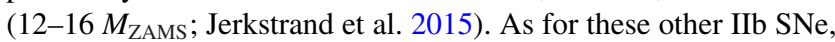
we find also for SN 2011fu that molecule formation is non-existent.

The extensive data set of SN 2011fu presented in this paper have permitted us to derive some of the characteristics of the ejecta of one more type IIb SN presenting a double-peaked LC, as well as to set constraints on some of its progenitor's properties. SN $2011 \mathrm{fu}$ is another example of a type IIb SN which shows evidence that the progenitor mass was in the range $13-18 \mathrm{M}_{\odot}$. This is in the higher range of stars that are seen to explode (Smartt 2015), which supports type IIb progenitor detections all being significantly more luminous than the II-P progenitors. Furthermore, as for all previously modelled IIb SNe, we determine a $M_{\text {ZAMS }}$ value much lower that what would have been needed for a wind-stripped WR progenitor, and evidence is piling up that most, if not all, type IIb $\mathrm{SNe}$ come from binary stripped progenitors.

\section{ACKNOWLEDGEMENTS}

AMG acknowledges financial support by the Spanish Ministerio de Economía y Competitividad (MINECO) grant ESP2013-41268-R. NER acknowledges the support from the European Union Seventh
Framework Programme (FP7/2007-2013) under grant agreement no. 267251 'Astronomy Fellowships in Italy' (AstroFIt). ST acknowledges support by TRR33 'The Dark Universe' of the German Research Foundation (DFG). AP, SB, NER, and LT are partially supported by the PRIN-INAF 2014 within the project 'Transient Universe: unveiling new types of stellar explosions with PESSTO.' This work was partly supported by the European Union FP7 programme through ERC grant number 320360. SJS acknowledges funding from the European Research Council under the European Union's Seventh Framework Programme (FP7/2007-2013)/ERC Grant agreement no. [291222] and STFC grants ST/I001123/1 and ST/L000709/1.

Data of this work have been taken in the framework of the European supernova collaboration involved in the ESO-NTT large programme 184.D-1140 led by Stefano Benetti.

This work is partially based on observations made with the Liverpool Telescope which is operated by Liverpool John Moores University with financial support from the UK Science and Technology Facilities Council, the Nordic Optical Telescope operated by the Nordic Optical Telescope Scientific Association, the Gran Telescopio de Canarias, and the William Hershel Telescope (operated by the Isaac Newton Group) in the Spanish Observatorio del Roque de los Muchachos (ORM) of the Instituto de Astrofísica de Canarias; the $2.2 \mathrm{~m}$ telescope of the Centro Astronómico Hispano Alemán (Calar Alto, Spain); the $1.82 \mathrm{~m}$ Copernico Telescope operated by INAF-Osservatorio Astronomico di Padova and the $1.22 \mathrm{~m}$ Galileo Telescope of Dipartimento di Fisica e Astonomia (Universitá di Padova) at the Asiago Observatory (Italy); the Telescopio Carlos Sánchez operated by the Instituto de Astrofísica de Canarias in the Spanish Observatorio del Teide; the Faulkes Telescope North situated on Mt. Haleakala (Hawaii, U.S.A.); and the $0.4 \mathrm{~m}$ telescope at the Observatorio Astronómico de Cantabria (Spain).

We would like to thank F. Ciabattari and E. Mazzoni from the ISSP for providing us the discovery and confirmation image of the $\mathrm{SN}$ taken at the Osservatorio di Monte Agliale (Lucca, Italy).

This research has made use of the NASA/IPAC Extragalactic Database (NED) which is operated by the Jet Propulsion Laboratory, California Institute of Technology, under contract with the National Aeronautics and Space Administration.

\section{REFERENCES}

Aldering G., Humphreys R. M., Richmond M., 1994, AJ, 107, 662

Barbon R., Benetti S., Cappellaro E., Patat F., Turatto M., Iijima T., 1995, A\&AS, 110,513

Ben-Ami S. et al., 2015, ApJ, 803, 40

Benvenuto O. G., Bersten M. C., Nomoto K., 2013, ApJ, 762, 74

Bersten M. C., Benvenuto O., Hamuy M., 2011, ApJ, 729, 61

Bersten M. C. et al., 2012, ApJ, 757, 31

Blinnikov S. I., Eastman R., Bartunov O. S., Popolitov V. A., Woosley S. E., 1998, ApJ, 496, 454

Bufano M. et al., 2014, MNRAS, 439, 1807

Cappellaro E., 2014, http://sngroup.oapd.inaf.it/snoopy.html

Chevalier R. A., Soderberg A. M., 2010, ApJ, 711, L40

Dessart L., Hillier D. J., 2005, A\&A, 439, 671

Ergon M. et al., 2014, A\&A, 562, A17

Ergon M. et al., 2015, A\&A, 580, A142

Filippenko A. V., 1988, AJ, 96, 1941

Folatelli G. et al., 2014, ApJ, 793, L22

Fox O. D., 2014, AJ, 790, 17

Gal-Yam et al., 2014, Nature, 509, 471

Groh J. H, 2014, A\&A, 572, L11

Hachinger S., Mazzali P. A., Taubenberger S., Hillebrandt W., Nomoto K., Sauer D. N., 2012, MNRAS, 422, 70 
Hamuy M. et al., 2009, AJ, 703, 1612

Heger A., Fryer C. L., Woosley S. E., Langer N., Hartmann D. H., 2003, ApJ, 591, 288

Inserra C. et al., 2013, A\&A, 555, A142

Jerkstrand A., Ergon M., Smartt S. J., Fransson C., Sollerman J., Taubenberger S., Bersten M., Spyromilio J., 2015, A\&A, 573, A12

Kumar B. et al., 2013, MNRAS, 431, 308

Lewis J. R. et al., 1994, MNRAS, 266, L27

Li W. et al., 2011, MNRAS, 412, 1441

Maeda K., Nomoto K., Mazzali P. A., Deng J., 2006, ApJ, 640, 854

Matthews K., Neugebauer G., Armus L., Soifer B. T., 2002, AJ, 123, 753

Maund J. R., Smartt S. J., 2009, Science, 324, 486

Maund J. R., Smartt S. J., Kudritzki R. P., Podsiadlowski P., Gilmore G. F., 2004, Nature, 427, 129

Maund J. R. et al., 2011, ApJ, 739, L37

Maurer I., Mazzali P. A., Taubenberger S., Hachinger S., 2010, MNRAS, 409,1441

Milisavljevic D., Fesen R. A., Gerardy C. L., Kirshner R. P., Challis P., 2010, ApJ, 709, 1343

Milisavljevic D. et al., 2013, ApJ, 767, 71

Modjaz M., Kirshner R. P., Blondin S., Challis P., Matheson T., 2008, ApJ, 687, L9

Modjaz M. et al., 2014, AJ, 147, 99

Morales-Garoffolo A. et al., 2014, MNRAS, 445, 1647

Mould J. R. et al., 2000, ApJ, 529, 786

Munari U., Zwitter T., 1997, A\&A, 318, 269

Nakar E., Piro A. L., 2014, ApJ, 788, 193

Nomoto K., Hashimoto M., 1988, Phys. Rep., 163, 13

Nomoto K., Suzuki T., Shigeyama T., Kumagai S., Yamaoka H., Saio H., 1993, Nature, 364, 507

Pastorello A. et al., 2008, MNRAS, 389, 955

Pignata G. et al., 2004, MNRAS, 355, 178
Podsiadlowski P., Hsu J. J. L., Joss P. C., Ross R. R., 1993, Nature, 364, 509

Poznanski D., Prochaska J. X., Bloom J. S., 2012, MNRAS, 426, 1465

Richmond M. W., Treffers R. R., Filippenko A. V., Paik Y., Leibundgut B., Schulman E., Cox C. V, 1994, AJ, 107, 1022

Schlafly E. F., Finkbeiner D. P., 2011, ApJ, 737, 103

Shigeyama T., Suzuki T., Kumagai S. Nomoto K., Saio H., Yamaoka H., 1994, ApJ, 420, 341

Shivvers I. et al., 2013, MNRAS, 436, 3614

Shivvers I., Groh J. H., Mauerhan J. C., Fox O. D., Leonard D. C., Filippenko A. V., 2015, ApJ, 806, 213

Skrutskie M. F. et al., 2006, AJ, 131, 1163

Smartt S. J., 2015, PASA, 32, 16

Smith N. et al., 2015, MNRAS, 449, 1876

Stritzinger M. et al., 2002, AJ, 124, 2100

Taubenberger S. et al., 2009, MNRAS, 397, 677

Taubenberger S. et al., 2011, MNRAS, 413, 2140

Tomasella L., Valenti S., Ochner P., Benetti S., Cappellaro E., Pastorello A., 2011, Central Bureau Electron. Telegrams, 2827, 2

Tsvetkov D. Y., Volkov I. M., Baklanov P., Blinnikov S., Tuchin O., 2009, Peremennye Zvezdy, 29, 2

Turatto M., Benetti S., Cappellaro E., 2003, in ESO Astrophysics Symposia, From Twilight to Highlight: The Physics of Supernovae, SpringerVerlag, Berlin, p. 200

Van Dyk S. D. et al., 2011, ApJ, 741, L28

Van Dyk S. D. et al., 2013, ApJ, 772, L32

Van Dyk S. D. et al., 2014, AJ, 147, 37

Woosley S. E., Eastman R. G., Weaver T. A., Pinto P. A., 1994, ApJ, 429, 300

Yaron O., Gal-Yam A., 2012, PASP, 124, 668

\section{APPENDIX A: INSTRUMENTAL SET-UP}

Table A1. Characteristics of the instruments used to acquire the photometric data of SN $2011 \mathrm{fu}$.

\begin{tabular}{|c|c|c|c|c|c|}
\hline Telescope & Instrument & Field of view & $\begin{array}{l}\text { Pixel scale } \\
\left.\text { (". pixel }{ }^{-1}\right)\end{array}$ & Filters & Key \\
\hline 2 m Liverpool & RatCam & $4^{\prime} 6 \times 4^{\prime} .6$ & 0.140 & $u B$ Vriz & LT \\
\hline $1.82 \mathrm{~m}$ Asiago & AFOSC & $8^{\prime} .1 \times 8^{\prime} .1$ & 0.473 & $U B V R i$ & A1p82 \\
\hline $2.2 \mathrm{~m}$ Calar Alto & CAFOS & $9^{\prime} \times 9^{\prime}$ & 0.530 & $U B V R I$ & $\mathrm{Ca} 2 \mathrm{p} 2$ \\
\hline $2.5 \mathrm{~m}$ Northern Optical & ALFOSC & $6^{\prime} .4 \times 6.4$ & 0.190 & $U B V R I$ & NOT \\
\hline $4.2 \mathrm{~m}$ William Herschel & ACAM & $9^{\prime} .0 \times 10^{\prime} .4$ & 0.250 & griz & WHT \\
\hline 2 m Faulkes North & fs02 & $10^{\prime} .5 \times 10^{\prime} .5$ & 0.304 & $B V R I$ & FTN1 \\
\hline 2 m Faulkes North & EM01 & $4.7 \times 4.7$ & 0.278 & $B V R I$ & FTN2 \\
\hline $0.6 \mathrm{~m}$ Esteve Duran & ST-9XE & $11^{\prime} .7 \times 11^{\prime} .7$ & 1.370 & $V I$ & TED \\
\hline $0.4 \mathrm{~m}$ Cantabria & ST-8XE & $24^{\prime} .0 \times 26^{\prime} .0$ & 0.900 & $V R I$ & $\mathrm{OAC}$ \\
\hline 0.36 m Celestron C14 & QHY9 CCD & $25^{\prime} .0 \times 19^{\prime} .0$ & 0.450 & Unfiltered & Xing Gao \\
\hline $0.5 \mathrm{~m}$ Newtonian & FLI Proline CCD & $20^{\prime} .4 \times 19^{\prime} .8$ & 2.320 & Unfiltered & F.Ciabattari/E.Mazzoni \\
\hline $0.92 \mathrm{~m}$ Asiago Schmidt & SCAM & $48^{\prime} .7 \times 48^{\prime} .7$ & 1.437 & BVRI & ASch \\
\hline 10.4 m Gran Telescopio Canarias & OSIRIS & $7.8 \times 8.5$ & 0.127 & gri & GTC \\
\hline 1.52 m Carlos Sánchez & CAIN3 & $4^{\prime} .2 \times 4^{\prime} .2$ & 1.000 & $J H K_{s}$ & TCS \\
\hline
\end{tabular}

Table A2. List of spectroscopic observations of SN $2011 \mathrm{fu}$.

\begin{tabular}{|c|c|c|c|c|c|c|}
\hline Date & $\begin{array}{c}\text { Julian date } \\
(+2400000.00)\end{array}$ & $\begin{array}{l}\text { Phase }^{a} \\
\text { (d) }\end{array}$ & Telescope+instrument & Grism+filter & $\begin{array}{c}\text { Range } \\
(\AA)\end{array}$ & Resolving power ${ }^{b}$ \\
\hline $2011 / 09 / 23$ & 55828.3 & 3.8 & AS1p82+AFOSC & $\mathrm{g} 4$ & $3500-8450$ & 300 \\
\hline $2011 / 09 / 24$ & 55828.5 & 4.0 & WHT+ISIS & $\mathrm{R} 158 \mathrm{R}+\mathrm{R} 300 \mathrm{~B}+\mathrm{gg} 495$ & $3200-10000$ & 1000,1300 \\
\hline $2011 / 09 / 25$ & 55830.4 & 5.9 & AS1p82+AFOSC & $\mathrm{g} 2+\mathrm{g} 4$ & $3500-10200$ & 200,300 \\
\hline $2011 / 09 / 26$ & 55831.3 & 6.8 & AS1p82+AFOSC & g4 & $3500-8450$ & 300 \\
\hline $2011 / 09 / 29$ & 55833.6 & 9.1 & NOT+ALFOSC & $\mathrm{g} 4$ & $3200-9100$ & 500 \\
\hline $2011 / 09 / 30$ & 55835.3 & 10.8 & AS1p82+AFOSC & $\mathrm{g} 2+\mathrm{g} 4$ & $3500-10200$ & 200,300 \\
\hline 2011/10/01 & 55836.4 & 11.9 & AS1p82+AFOSC & g4 & $3500-8450$ & 300 \\
\hline $2011 / 10 / 03$ & 55838.5 & 14.0 & AS1p82+AFOSC & $\mathrm{g} 4$ & $3500-8450$ & 300 \\
\hline 2011/10/06 & 55841.4 & 16.9 & $\mathrm{Ca} 2 \mathrm{p} 2+\mathrm{CAFOS}$ & $\mathrm{B} 200+\mathrm{R} 200$ & $3200-11000$ & 500,500 \\
\hline
\end{tabular}


Table A2. - continued.

\begin{tabular}{|c|c|c|c|c|c|c|}
\hline Date & $\begin{array}{c}\text { Julian date } \\
(+2400000.00)\end{array}$ & $\begin{array}{l}\text { Phase }^{a} \\
\text { (d) }\end{array}$ & Telescope+instrument & Grism+filter & $\begin{array}{c}\text { Range } \\
(\AA)\end{array}$ & Resolving power ${ }^{b}$ \\
\hline $2011 / 10 / 10$ & 55844.6 & 20.1 & $\mathrm{NOT}+\mathrm{ALFOSC}$ & g4 & $3200-9100$ & 500 \\
\hline 2011/10/17 & 55851.5 & 27.0 & $\mathrm{Ca} 2 \mathrm{p} 2+\mathrm{CAFOS}$ & $\mathrm{B} 200+\mathrm{R} 200$ & $3200-11000$ & 500,500 \\
\hline 2011/10/17 & 55851.6 & 27.1 & WHT+ACAM & V400 & $3500-9400$ & 500 \\
\hline $2011 / 10 / 24$ & 55858.5 & 34.0 & AS1p82+AFOSC & $\mathrm{g} 4$ & $3500-8450$ & 300 \\
\hline $2011 / 10 / 27$ & 55862.4 & 37.9 & NOT+ALFOSC & g4 & $3200-9100$ & 500 \\
\hline $2011 / 10 / 30$ & 55864.6 & 40.1 & AS1p82+AFOSC & g4 & $3500-8450$ & 300 \\
\hline $2011 / 10 / 31$ & 55866.4 & 41.9 & AS1p $82+$ AFOSC & g4 & $3500-8450$ & 300 \\
\hline 2011/11/01 & 55867.4 & 42.9 & $\mathrm{Ca} 2 \mathrm{p} 2+\mathrm{CAFOS}$ & $\mathrm{G} 200$ & $3700-9500$ & 500 \\
\hline 2011/11/07 & 55872.5 & 48.0 & WHT+ISIS & $\mathrm{R} 158 \mathrm{R}+\mathrm{R} 300 \mathrm{~B}+\mathrm{gg} 495$ & $3200-10000$ & 1000,1300 \\
\hline $2011 / 11 / 15$ & 55881.4 & 56.9 & $\mathrm{Ca} 2 \mathrm{p} 2+\mathrm{CAFOS}$ & G200 & $3700-9500$ & 500 \\
\hline $2011 / 11 / 20$ & 55886.4 & 61.9 & AS1p82+AFOSC & g4 & $3500-8450$ & 300 \\
\hline $2011 / 11 / 29$ & 55894.5 & 70.0 & NOT+ALFOSC & $\mathrm{g} 4$ & $3200-9100$ & 500 \\
\hline $2011 / 11 / 29$ & 55895.4 & 70.9 & $\mathrm{Ca} 2 \mathrm{p} 2+\mathrm{CAFOS}$ & R200 & $6300-11000$ & 500 \\
\hline $2011 / 12 / 17$ & 55913.5 & 89.0 & TNG+DOLORES & $\mathrm{LRR}+\mathrm{LRB}$ & $3000-10000$ & 460,460 \\
\hline $2011 / 12 / 20$ & 55916.4 & 91.9 & AS1p82+AFOSC & g4 & $3500-8450$ & 300 \\
\hline $2011 / 12 / 21$ & 55917.4 & 92.9 & NOT+ALFOSC & $\mathrm{g} 4+\mathrm{g} 5$ & $3200-10250$ & 500,500 \\
\hline $2012 / 01 / 01$ & 55928.4 & 103.9 & $\mathrm{Ca} 2 \mathrm{p} 2+\mathrm{CAFOS}$ & $\mathrm{G} 200+\mathrm{gg} 495$ & $4800-9500$ & 500 \\
\hline $2012 / 02 / 22$ & 55980.3 & 155.8 & AS1p82+AFOSC & $\mathrm{g} 4$ & $3500-8450$ & 300 \\
\hline $2012 / 07 / 20$ & 56128.6 & 304.1 & GTC+OSIRIS & $\mathrm{R} 300 \mathrm{~B}+\mathrm{R} 300 \mathrm{R}$ & $3600-10000$ & 430 \\
\hline
\end{tabular}

Notes. ${ }^{a}$ Phase with respect to our adopted explosion epoch JD $=2455824.5 \pm 0.7$.

${ }^{b}$ The resolution was measured from the full width at half-maximum of the night sky lines. The resolving power values reported here are for a reference wavelength of $6500 \AA$. 


\section{APPENDIX B: SN 2011FU'S PHOTOMETRIC DATA}

Table B1. Optical Bessell photometry of SN 2011fu. The $U$ data are not S-corrected, note that the reported RI LT data are the S-corrected Sloan $r i$ magnitudes.

\begin{tabular}{|c|c|c|c|c|c|c|c|c|}
\hline Date & $\begin{array}{c}\text { JD } \\
(+2400000.00)\end{array}$ & $\begin{array}{l}\text { Phase }^{a} \\
\text { (d) }\end{array}$ & $\begin{array}{c}U \\
(\mathrm{mag})\end{array}$ & $\begin{array}{c}B \\
(\mathrm{mag})\end{array}$ & $\begin{array}{c}V \\
(\mathrm{mag})\end{array}$ & $\begin{array}{c}R \\
(\mathrm{mag})\end{array}$ & $\begin{array}{c}I \\
(\mathrm{mag})\end{array}$ & Key \\
\hline $2011 / 09 / 20$ & 55825.2 & 0.7 & - & - & $16.32 \pm 0.14$ & - & - & Xing Gao \\
\hline $2011 / 09 / 21$ & 55825.5 & 1.0 & - & - & - & $16.07 \pm 0.13$ & - & F. Ciabattari/E.Mazzoni \\
\hline $2011 / 09 / 21$ & 55826.4 & 1.9 & - & - & $16.21 \pm 0.15$ & - & - & Xing Gao \\
\hline $2011 / 09 / 21$ & 55826.4 & 1.9 & - & - & - & $16.01 \pm 0.08$ & - & F. Ciabattari/E.Mazzoni \\
\hline $2011 / 09 / 23$ & 55828.3 & 3.8 & - & - & - & $16.27 \pm 0.02$ & - & A1p82 \\
\hline $2011 / 09 / 23$ & 55828.4 & 3.9 & - & - & $16.45 \pm 0.24$ & - & - & Xing Gao \\
\hline $2011 / 09 / 25$ & 55830.5 & 6.0 & $16.52 \pm 0.02$ & $17.10 \pm 0.02$ & $16.83 \pm 0.01$ & $16.65 \pm 0.02$ & $16.52 \pm 0.02$ & A1p82 \\
\hline $2011 / 09 / 26$ & 55831.2 & 6.7 & - & - & $17.09 \pm 0.15$ & - & - & Xing Gao \\
\hline $2011 / 09 / 26$ & 55831.4 & 6.9 & $16.43 \pm 0.03$ & $17.31 \pm 0.02$ & $17.02 \pm 0.01$ & $16.80 \pm 0.02$ & $16.62 \pm 0.02$ & A1p82 \\
\hline $2011 / 09 / 27$ & 55831.5 & 7.0 & - & $17.40 \pm 0.02$ & $17.19 \pm 0.01$ & $16.78 \pm 0.04$ & $16.92 \pm 0.03$ & LT \\
\hline $2011 / 09 / 29$ & 55833.5 & 9.0 & - & $17.85 \pm 0.02$ & $17.41 \pm 0.03$ & $17.12 \pm 0.04$ & $16.84 \pm 0.03$ & LT \\
\hline $2011 / 09 / 29$ & 55834.5 & 10.0 & $17.86 \pm 0.03$ & $17.93 \pm 0.02$ & $17.57 \pm 0.01$ & $17.28 \pm 0.02$ & $17.00 \pm 0.02$ & A1p 82 \\
\hline $2011 / 09 / 29$ & 55834.6 & 10.1 & - & $17.91 \pm 0.02$ & $17.55 \pm 0.02$ & $17.11 \pm 0.02$ & $16.87 \pm 0.03$ & LT \\
\hline $2011 / 09 / 30$ & 55835.3 & 10.8 & - & - & $17.40 \pm 0.14$ & - & - & Xing Gao \\
\hline $2011 / 09 / 30$ & 55835.4 & 10.9 & - & $17.97 \pm 0.02$ & $17.55 \pm 0.02$ & $17.24 \pm 0.01$ & $16.98 \pm 0.05$ & A1p82 \\
\hline 2011/10/01 & 55835.5 & 11.0 & - & $17.95 \pm 0.01$ & $17.55 \pm 0.03$ & $17.08 \pm 0.03$ & $17.02 \pm 0.03$ & LT \\
\hline 2011/10/01 & 55836.4 & 11.9 & $17.74 \pm 0.06$ & $17.96 \pm 0.02$ & $17.51 \pm 0.01$ & $17.19 \pm 0.01$ & $17.02 \pm 0.03$ & A1p 82 \\
\hline 2011/10/03 & 55837.5 & 13.0 & - & $17.91 \pm 0.02$ & $17.46 \pm 0.03$ & $17.07 \pm 0.43$ & $16.83 \pm 0.05$ & LT \\
\hline 2011/10/03 & 55838.5 & 14.0 & $17.96 \pm 0.06$ & $17.84 \pm 0.02$ & $17.37 \pm 0.01$ & $17.06 \pm 0.01$ & $16.90 \pm 0.03$ & A1p82 \\
\hline $2011 / 10 / 05$ & 55840.2 & 15.7 & - & - & $17.34 \pm 0.18$ & - & - & Xing Gao \\
\hline 2011/10/06 & 55840.5 & 16.0 & - & $17.79 \pm 0.02$ & $17.26 \pm 0.02$ & $16.91 \pm 0.03$ & $16.64 \pm 0.04$ & LT \\
\hline 2011/10/06 & 55841.4 & 16.9 & $17.45 \pm 0.04$ & $17.64 \pm 0.03$ & $17.17 \pm 0.02$ & $16.78 \pm 0.02$ & $16.71 \pm 0.06$ & $\mathrm{Ca} 2 \mathrm{p} 2$ \\
\hline $2011 / 10 / 07$ & 55841.5 & 17.0 & - & $17.60 \pm 0.03$ & $17.24 \pm 0.05$ & $16.77 \pm 0.06$ & $16.54 \pm 0.05$ & LT \\
\hline 2011/10/08 & 55842.5 & 18.0 & - & - & $17.08 \pm 0.02$ & - & - & LT \\
\hline $2011 / 10 / 08$ & 55843.3 & 18.8 & - & - & $16.96 \pm 0.15$ & - & - & Xing Gao \\
\hline $2011 / 10 / 10$ & 55844.5 & 20.0 & - & $17.58 \pm 0.02$ & $16.99 \pm 0.03$ & $16.64 \pm 0.03$ & $16.46 \pm 0.03$ & LT \\
\hline $2011 / 10 / 10$ & 55844.5 & 20.0 & - & - & $16.90 \pm 0.06$ & - & $16.66 \pm 0.05$ & TED \\
\hline 2011/10/11 & 55845.5 & 21.0 & - & $17.60 \pm 0.04$ & $16.97 \pm 0.03$ & $16.61 \pm 0.07$ & $16.40 \pm 0.05$ & LT \\
\hline 2011/10/11 & 55845.5 & 21.0 & - & - & $16.94 \pm 0.04$ & $16.62 \pm 0.03$ & - & $\mathrm{OAC}$ \\
\hline 2011/10/11 & 55845.5 & 21.0 & - & - & $16.94 \pm 0.02$ & - & $16.56 \pm 0.03$ & TED \\
\hline $2011 / 10 / 12$ & 55846.5 & 22.0 & - & $17.47 \pm 0.03$ & $16.92 \pm 0.04$ & $16.57 \pm 0.03$ & $16.39 \pm 0.03$ & LT \\
\hline 2011/10/12 & 55846.5 & 22.0 & - & - & - & $16.58 \pm 0.05$ & $16.50 \pm 0.06$ & OAC \\
\hline $2011 / 10 / 13$ & 55847.5 & 23.0 & - & $17.53 \pm 0.04$ & $16.90 \pm 0.04$ & $16.54 \pm 0.04$ & $16.45 \pm 0.03$ & $\mathrm{LT}$ \\
\hline $2011 / 10 / 14$ & 55848.5 & 24.0 & - & $17.58 \pm 0.07$ & $16.92 \pm 0.06$ & $16.52 \pm 0.06$ & $16.36 \pm 0.04$ & $\mathrm{LT}$ \\
\hline $2011 / 10 / 15$ & 55849.5 & 25.0 & - & $17.73 \pm 0.03$ & $16.96 \pm 0.03$ & $16.54 \pm 0.06$ & $16.37 \pm 0.04$ & LT \\
\hline 2011/10/16 & 55851.4 & 26.9 & - & - & $17.07 \pm 0.07$ & - & $16.56 \pm 0.04$ & TED \\
\hline 2011/10/16 & 55851.5 & 27.0 & $18.12 \pm 0.10$ & $17.87 \pm 0.03$ & $17.08 \pm 0.02$ & $16.66 \pm 0.02$ & $16.56 \pm 0.04$ & $\mathrm{Ca} 2 \mathrm{p} 2$ \\
\hline $2011 / 10 / 18$ & 55852.5 & 28.0 & - & $18.05 \pm 0.02$ & $17.14 \pm 0.02$ & $16.63 \pm 0.03$ & $16.44 \pm 0.03$ & LT \\
\hline 2011/10/18 & 55853.2 & 28.7 & - & - & $17.30 \pm 0.13$ & - & - & Xing Gao \\
\hline $2011 / 10 / 18$ & 55853.4 & 28.9 & - & - & $17.24 \pm 0.07$ & - & $16.53 \pm 0.04$ & TED \\
\hline $2011 / 10 / 22$ & 55857.2 & 32.7 & - & - & $17.43 \pm 0.12$ & - & - & Xing Gao \\
\hline $2011 / 10 / 23$ & 55857.6 & 33.1 & - & $18.72 \pm 0.03$ & $17.57 \pm 0.03$ & $16.83 \pm 0.06$ & $16.59 \pm 0.04$ & LT \\
\hline $2011 / 10 / 23$ & 55858.2 & 33.7 & - & - & $17.41 \pm 0.14$ & - & - & Xing Gao \\
\hline $2011 / 10 / 24$ & 55858.6 & 34.1 & - & $18.70 \pm 0.02$ & $17.61 \pm 0.01$ & $16.98 \pm 0.01$ & $16.73 \pm 0.03$ & A1p82 \\
\hline $2011 / 10 / 24$ & 55859.2 & 34.7 & - & - & $17.47 \pm 0.13$ & - & - & Xing Gao \\
\hline $2011 / 10 / 25$ & 55859.5 & 35.0 & - & $18.78 \pm 0.04$ & $17.63 \pm 0.03$ & $16.92 \pm 0.06$ & $16.70 \pm 0.04$ & LT \\
\hline $2011 / 10 / 25$ & 55860.4 & 35.9 & - & - & $17.79 \pm 0.05$ & - & $16.89 \pm 0.04$ & TED \\
\hline $2011 / 10 / 26$ & 55860.5 & 36.0 & - & $18.81 \pm 0.04$ & $17.68 \pm 0.03$ & $16.97 \pm 0.06$ & $16.67 \pm 0.04$ & LT \\
\hline $2011 / 10 / 27$ & 55861.5 & 37.0 & - & $18.91 \pm 0.04$ & $17.79 \pm 0.03$ & $16.99 \pm 0.06$ & $17.08 \pm 0.04$ & LT \\
\hline $2011 / 10 / 28$ & 55862.5 & 38.0 & $19.70 \pm 0.05$ & $19.10 \pm 0.03$ & $17.77 \pm 0.02$ & $17.13 \pm 0.02$ & $16.64 \pm 0.01$ & NOT \\
\hline $2011 / 10 / 28$ & 55862.5 & 38.0 & - & $19.08 \pm 0.04$ & $17.80 \pm 0.03$ & $17.21 \pm 0.02$ & $16.76 \pm 0.02$ & LT \\
\hline $2011 / 10 / 29$ & 55863.5 & 39.0 & - & $19.06 \pm 0.04$ & $17.83 \pm 0.03$ & $17.14 \pm 0.06$ & $16.74 \pm 0.04$ & $\mathrm{LT}$ \\
\hline $2011 / 10 / 30$ & 55864.5 & 40.0 & - & $19.07 \pm 0.07$ & $17.80 \pm 0.05$ & $17.26 \pm 0.06$ & - & LT \\
\hline $2011 / 10 / 30$ & 55864.5 & 40.0 & - & $18.97 \pm 0.06$ & $17.89 \pm 0.03$ & $17.20 \pm 0.03$ & $17.01 \pm 0.03$ & A1p 82 \\
\hline $2011 / 10 / 31$ & 55866.5 & 42.0 & - & $19.10 \pm 0.06$ & $17.97 \pm 0.03$ & $17.31 \pm 0.02$ & $17.03 \pm 0.03$ & A1p82 \\
\hline 2011/11/01 & 55866.6 & 42.1 & - & $19.17 \pm 0.06$ & $17.99 \pm 0.05$ & $17.20 \pm 0.03$ & $16.90 \pm 0.02$ & LT \\
\hline
\end{tabular}


Table B1. - continued

\begin{tabular}{|c|c|c|c|c|c|c|c|c|}
\hline Date & $\begin{array}{c}\text { JD } \\
(+2400000.00)\end{array}$ & $\begin{array}{l}\text { Phase }^{a} \\
\text { (d) }\end{array}$ & $\begin{array}{c}U \\
(\mathrm{mag})\end{array}$ & $\begin{array}{c}B \\
(\mathrm{mag})\end{array}$ & $\begin{array}{c}V \\
(\mathrm{mag})\end{array}$ & $\begin{array}{c}R \\
(\mathrm{mag})\end{array}$ & $\begin{array}{c}I \\
(\mathrm{mag})\end{array}$ & Key \\
\hline 2011/11/01 & 55867.4 & 42.9 & $20.02 \pm 0.10$ & $19.06 \pm 0.02$ & $18.03 \pm 0.02$ & $17.23 \pm 0.02$ & $17.12 \pm 0.05$ & $\mathrm{Ca} 2 \mathrm{p} 2$ \\
\hline 2011/11/05 & 55871.1 & 46.6 & - & - & $17.88 \pm 0.17$ & - & - & Xing Gao \\
\hline 2011/11/06 & 55872.1 & 47.6 & - & - & $18.00 \pm 0.15$ & - & - & Xing Gao \\
\hline 2011/11/07 & 55872.6 & 48.1 & - & - & - & $17.57 \pm 0.18$ & $17.15 \pm 0.03$ & WHT \\
\hline 2011/11/11 & 55876.9 & 52.4 & - & - & - & $17.59 \pm 0.03$ & $17.30 \pm 0.03$ & FTN1 \\
\hline 2011/11/14 & 55880.2 & 55.7 & - & $19.41 \pm 0.10$ & $18.27 \pm 0.10$ & $17.42 \pm 0.09$ & $17.32 \pm 0.09$ & ASch \\
\hline 2011/11/15 & 55881.4 & 56.9 & - & $19.29 \pm 0.04$ & $18.25 \pm 0.03$ & $17.65 \pm 0.02$ & $17.42 \pm 0.02$ & $\mathrm{Ca} 2 \mathrm{p} 2$ \\
\hline 2011/11/17 & 55882.9 & 48.4 & - & $19.51 \pm 0.06$ & $18.28 \pm 0.05$ & $17.78 \pm 0.03$ & $17.43 \pm 0.03$ & FTN1 \\
\hline 2011/11/19 & 55885.4 & 60.9 & - & $19.49 \pm 0.04$ & $18.49 \pm 0.02$ & $17.82 \pm 0.02$ & $17.45 \pm 0.03$ & A1p82 \\
\hline 2011/11/21 & 55886.5 & 62.0 & - & $19.64 \pm 0.05$ & $18.56 \pm 0.03$ & $17.76 \pm 0.04$ & $17.39 \pm 0.02$ & A1p82 \\
\hline $2011 / 11 / 22$ & 55888.1 & 63.6 & - & - & $18.38 \pm 0.13$ & - & - & Xing Gao \\
\hline 2011/11/23 & 55888.5 & 64.0 & - & $19.47 \pm 0.05$ & $18.40 \pm 0.06$ & $17.67 \pm 0.04$ & $17.42 \pm 0.05$ & LT \\
\hline $2011 / 11 / 25$ & 55890.8 & 66.3 & - & $19.66 \pm 0.06$ & $18.45 \pm 0.05$ & $17.80 \pm 0.03$ & $17.57 \pm 0.03$ & FTN1 \\
\hline $2011 / 11 / 27$ & 55893.0 & 68.5 & - & - & $18.41 \pm 0.11$ & $17.78 \pm 0.13$ & $17.59 \pm 0.13$ & FTN1 \\
\hline 2011/11/29 & 55894.9 & 70.4 & - & $19.52 \pm 0.07$ & $18.50 \pm 0.05$ & $17.94 \pm 0.04$ & $17.66 \pm 0.03$ & FTN1 \\
\hline $2011 / 11 / 29$ & 55894.9 & 70.4 & - & $19.68 \pm 0.03$ & $18.59 \pm 0.02$ & $17.96 \pm 0.02$ & $17.46 \pm 0.01$ & NOT \\
\hline $2011 / 11 / 30$ & 55896.4 & 71.9 & - & $19.63 \pm 0.05$ & $18.57 \pm 0.06$ & $17.89 \pm 0.05$ & $17.55 \pm 0.06$ & LT \\
\hline 2011/12/01 & 55896.8 & 72.3 & - & - & $18.53 \pm 0.05$ & $17.97 \pm 0.04$ & $17.71 \pm 0.03$ & FTN1 \\
\hline 2011/12/06 & 55901.8 & 77.3 & - & $19.91 \pm 0.06$ & $18.56 \pm 0.05$ & $18.04 \pm 0.03$ & $17.77 \pm 0.03$ & FTN2 \\
\hline 2011/12/12 & 55908.4 & 83.9 & - & - & $18.87 \pm 0.07$ & $18.09 \pm 0.05$ & $17.73 \pm 0.01$ & LT \\
\hline $2011 / 12 / 20$ & 55916.4 & 91.9 & - & $19.71 \pm 0.09$ & $18.94 \pm 0.09$ & $18.34 \pm 0.04$ & $17.97 \pm 0.05$ & A1p82 \\
\hline $2011 / 12 / 22$ & 55917.8 & 93.3 & $20.01 \pm 0.07$ & $19.80 \pm 0.03$ & $18.88 \pm 0.03$ & $18.38 \pm 0.03$ & $17.96 \pm 0.06$ & NOT \\
\hline $2011 / 12 / 22$ & 55917.8 & 93.3 & - & $19.75 \pm 0.06$ & $18.85 \pm 0.05$ & $18.40 \pm 0.03$ & $18.11 \pm 0.03$ & FTN2 \\
\hline 2012/01/01 & 55928.3 & 103.8 & - & $19.80 \pm 0.02$ & $19.07 \pm 0.03$ & $18.52 \pm 0.03$ & $18.34 \pm 0.05$ & $\mathrm{Ca} 2 \mathrm{p} 2$ \\
\hline 2012/01/09 & 55936.5 & 112.0 & - & $19.96 \pm 0.08$ & $19.18 \pm 0.07$ & $18.72 \pm 0.05$ & $18.44 \pm 0.05$ & LT \\
\hline 2012/01/12 & 55939.4 & 114.9 & - & $19.92 \pm 0.03$ & $19.33 \pm 0.03$ & $18.85 \pm 0.02$ & $18.67 \pm 0.05$ & $\mathrm{Ca} 2 \mathrm{p} 2$ \\
\hline 2012/01/15 & 55942.3 & 117.8 & - & $20.00 \pm 0.08$ & $19.24 \pm 0.07$ & $18.76 \pm 0.06$ & $18.41 \pm 0.06$ & $\mathrm{LT}$ \\
\hline 2012/01/27 & 55954.4 & 129.9 & - & $20.32 \pm 0.08$ & $19.48 \pm 0.07$ & $19.04 \pm 0.06$ & $18.80 \pm 0.04$ & LT \\
\hline 2012/02/17 & 55975.3 & 150.8 & - & $20.55 \pm 0.13$ & $19.98 \pm 0.09$ & $19.60 \pm 0.06$ & $19.19 \pm 0.05$ & A1p82 \\
\hline $2012 / 02 / 22$ & 55980.3 & 155.8 & - & $20.69 \pm 0.18$ & $19.99 \pm 0.15$ & $19.61 \pm 0.04$ & $19.01 \pm 0.04$ & A1p82 \\
\hline $2012 / 02 / 23$ & 55981.4 & 156.9 & - & - & $20.13 \pm 0.08$ & $19.73 \pm 0.03$ & $19.27 \pm 0.08$ & LT \\
\hline $2012 / 02 / 25$ & 55983.4 & 148.9 & - & - & $19.89 \pm 0.25$ & $19.48 \pm 0.11$ & $19.24 \pm 0.13$ & LT \\
\hline $2012 / 07 / 20$ & 56128.6 & 304.1 & - & - & - & $21.54 \pm 0.02$ & $21.45 \pm 0.04$ & GTC \\
\hline
\end{tabular}

Note. ${ }^{a}$ Phase in days with respect to the adopted explosion date $\mathrm{JD}=2455824.5 \pm 0.7$. 
Table B2. Optical Sloan photometry of SN 2011fu in the Vega system.

\begin{tabular}{|c|c|c|c|c|c|c|}
\hline Date & $\begin{array}{c}\text { JD } \\
(+2400000.00)\end{array}$ & $\begin{array}{l}\text { Phase }^{a} \\
\text { (d) }\end{array}$ & $\begin{array}{c}u \\
(\mathrm{mag})\end{array}$ & $\begin{array}{c}g \\
(\mathrm{mag})\end{array}$ & $\begin{array}{c}z \\
(\mathrm{mag})\end{array}$ & Key \\
\hline $2011 / 09 / 27$ & 55831.5 & 7.0 & $16.43 \pm 0.06$ & - & $16.37 \pm 0.03$ & LT \\
\hline $2011 / 09 / 29$ & 55833.5 & 9.0 & $17.02 \pm 0.04$ & - & $16.61 \pm 0.04$ & LT \\
\hline $2011 / 09 / 30$ & 55834.6 & 10.1 & $17.37 \pm 0.06$ & - & $16.77 \pm 0.03$ & LT \\
\hline $2011 / 10 / 01$ & 55835.5 & 11.0 & $17.45 \pm 0.09$ & - & $16.70 \pm 0.03$ & LT \\
\hline $2011 / 10 / 03$ & 55837.5 & 13.0 & - & - & $16.66 \pm 0.05$ & LT \\
\hline $2011 / 10 / 06$ & 55840.5 & 16.0 & $17.38 \pm 0.04$ & - & $16.49 \pm 0.04$ & $\mathrm{LT}$ \\
\hline 2011/10/08 & 55842.5 & 18.0 & - & - & $16.40 \pm 0.06$ & LT \\
\hline $2011 / 10 / 10$ & 55844.5 & 20.0 & $17.23 \pm 0.03$ & - & $16.32 \pm 0.03$ & LT \\
\hline 2011/10/11 & 55845.5 & 21.0 & $17.5 \pm 0.13$ & - & $16.28 \pm 0.05$ & LT \\
\hline $2011 / 10 / 12$ & 55846.5 & 22.0 & $17.71 \pm 0.11$ & - & $16.30 \pm 0.03$ & LT \\
\hline $2011 / 10 / 13$ & 55847.5 & 25.0 & $17.75 \pm 0.03$ & - & $16.34 \pm 0.05$ & LT \\
\hline $2011 / 10 / 14$ & 55848.5 & 24.0 & $17.74 \pm 0.11$ & - & $16.36 \pm 0.05$ & LT \\
\hline $2011 / 10 / 15$ & 55849.5 & 25.0 & $17.82 \pm 0.10$ & - & $16.22 \pm 0.05$ & LT \\
\hline $2011 / 10 / 18$ & 55852.5 & 28.0 & $18.22 \pm 0.10$ & - & $16.32 \pm 0.03$ & LT \\
\hline $2011 / 10 / 23$ & 55857.6 & 33.1 & - & - & $16.46 \pm 0.05$ & LT \\
\hline $2011 / 10 / 25$ & 55859.5 & 35.0 & - & - & $16.54 \pm 0.05$ & LT \\
\hline $2011 / 10 / 26$ & 55860.5 & 36.0 & - & - & $16.52 \pm 0.05$ & LT \\
\hline $2011 / 10 / 27$ & 55861.5 & 37.0 & - & - & $16.59 \pm 0.05$ & LT \\
\hline $2011 / 10 / 28$ & 55862.5 & 38.0 & $19.37 \pm 0.03$ & - & $16.66 \pm 0.02$ & $\mathrm{LT}$ \\
\hline $2011 / 10 / 29$ & 55863.5 & 39.0 & $19.24 \pm 0.07$ & - & $16.65 \pm 0.04$ & LT \\
\hline $2011 / 10 / 30$ & 55864.5 & 40.0 & - & - & $16.75 \pm 0.04$ & LT \\
\hline $2011 / 11 / 01$ & 55866.6 & 42.1 & - & - & $16.69 \pm 0.02$ & LT \\
\hline $2011 / 11 / 07$ & 55872.6 & 48.1 & - & $18.72 \pm 0.29$ & $16.85 \pm 0.05$ & WHT \\
\hline $2011 / 11 / 23$ & 55888.5 & 64.0 & - & - & $17.10 \pm 0.06$ & LT \\
\hline $2011 / 11 / 30$ & 55896.4 & 71.9 & - & - & $17.35 \pm 0.05$ & $\mathrm{LT}$ \\
\hline $2011 / 12 / 12$ & 55908.4 & 83.9 & - & - & $17.51 \pm 0.05$ & LT \\
\hline $2012 / 01 / 09$ & 55936.5 & 112.0 & - & - & $18.07 \pm 0.07$ & LT \\
\hline $2012 / 01 / 15$ & 55942.3 & 117.8 & - & - & $18.14 \pm 0.08$ & LT \\
\hline $2012 / 01 / 27$ & 55954.4 & 129.9 & - & - & $18.25 \pm 0.07$ & LT \\
\hline $2012 / 02 / 23$ & 55981.4 & 156.9 & - & - & $18.82 \pm 0.16$ & LT \\
\hline $2012 / 07 / 20$ & 56128.6 & 304.1 & - & $22.41 \pm 0.15$ & - & GTC \\
\hline
\end{tabular}

Note. ${ }^{a}$ Phase in days with respect to the adopted explosion date $\mathrm{JD}=2455824.5 \pm 0.7$.

Table B3. NIR photometry of SN 2011 fu.

\begin{tabular}{lcccccc}
\hline Date & $\begin{array}{c}\text { JD } \\
(+2400000.00)\end{array}$ & $\begin{array}{c}\text { Phase }^{a} \\
(\mathrm{~d})\end{array}$ & $\begin{array}{c}J \\
(\mathrm{mag})\end{array}$ & $\begin{array}{c}H \\
(\mathrm{mag})\end{array}$ & $\begin{array}{c}K_{\mathrm{S}} \\
(\mathrm{mag})\end{array}$ & Key \\
\hline $2011 / 10 / 06$ & 55840.6 & 16.1 & $16.20 \pm 0.12$ & $16.10 \pm 0.14$ & $15.75 \pm 0.14$ & TCS \\
$2011 / 10 / 10$ & 55844.6 & 20.1 & $16.08 \pm 0.12$ & - & $15.72 \pm 0.27$ & TCS \\
$2011 / 10 / 12$ & 55847.4 & 23.0 & $16.15 \pm 0.08$ & $15.70 \pm 0.15$ & $15.63 \pm 0.34$ & TCS \\
$2011 / 10 / 29$ & 55864.5 & 40.0 & $16.28 \pm 0.13$ & - & - & TCS \\
$2011 / 10 / 31$ & 55866.5 & 42.0 & $16.54 \pm 0.15$ & $16.06 \pm 0.15$ & $15.77 \pm 0.21$ & TCS \\
$2011 / 11 / 04$ & 55869.5 & 45.0 & $16.74 \pm 0.15$ & $16.27 \pm 0.12$ & $16.17 \pm 0.27$ & TCS \\
\hline
\end{tabular}

Note. ${ }^{a}$ Phase in days with respect to the adopted explosion date JD $=2455824.5 \pm 0.7$. 


\section{A. Morales-Garoffolo et al.}

Table B4. Johnson-Cousins optical and 2MASS NIR magnitudes and associated errors for the stellar sequence used in the calibration process of SN 2011fu's photometry.

\begin{tabular}{|c|c|c|c|c|c|c|c|c|}
\hline Star & $\begin{array}{c}U \\
(\mathrm{mag})\end{array}$ & $\begin{array}{c}B \\
(\mathrm{mag})\end{array}$ & $\begin{array}{c}V \\
(\mathrm{mag})\end{array}$ & $\begin{array}{c}R \\
(\mathrm{mag})\end{array}$ & $\begin{array}{c}I \\
(\mathrm{mag})\end{array}$ & $\begin{array}{c}J \\
(\mathrm{mag})\end{array}$ & $\begin{array}{c}H \\
(\mathrm{mag})\end{array}$ & $\begin{array}{c}K_{\mathrm{s}} \\
(\mathrm{mag})\end{array}$ \\
\hline 1 & $19.26 \pm 0.10$ & $18.66 \pm 0.02$ & $17.74 \pm 0.02$ & $17.20 \pm 0.03$ & $16.65 \pm 0.03$ & - & - & - \\
\hline 2 & $18.25 \pm 0.10$ & $18.17 \pm 0.02$ & $17.52 \pm 0.02$ & $17.10 \pm 0.05$ & $16.79 \pm 0.03$ & - & - & - \\
\hline 3 & $18.26 \pm 0.06$ & $18.25 \pm 0.02$ & $17.55 \pm 0.02$ & $17.24 \pm 0.02$ & $16.87 \pm 0.03$ & $16.24 \pm 0.12$ & $15.96 \pm 0.19$ & $15.79 \pm 0.26$ \\
\hline 4 & $18.98 \pm 0.09$ & $18.14 \pm 0.02$ & $17.18 \pm 0.02$ & $16.63 \pm 0.02$ & $16.14 \pm 0.03$ & $15.45 \pm 0.06$ & $14.81 \pm 0.08$ & $14.81 \pm 0.11$ \\
\hline 5 & $17.75 \pm 0.04$ & $17.83 \pm 0.02$ & $17.25 \pm 0.02$ & $16.90 \pm 0.02$ & $16.58 \pm 0.02$ & $15.91 \pm 0.08$ & $15.77 \pm 0.15$ & $15.51 \pm 0.20$ \\
\hline 6 & $15.68 \pm 0.09$ & $15.51 \pm 0.02$ & $14.88 \pm 0.07$ & $14.47 \pm 0.07$ & $14.11 \pm 0.06$ & $13.49 \pm 0.02$ & $13.16 \pm 0.03$ & $13.07 \pm 0.03$ \\
\hline 7 & $17.88 \pm 0.04$ & $17.77 \pm 0.02$ & $17.14 \pm 0.03$ & $16.77 \pm 0.03$ & $16.41 \pm 0.02$ & $15.83 \pm 0.08$ & $15.30 \pm 0.10$ & $15.42 \pm 0.19$ \\
\hline 8 & $19.94 \pm 0.05$ & $18.82 \pm 0.02$ & $17.58 \pm 0.03$ & $16.82 \pm 0.02$ & $16.15 \pm 0.03$ & $15.11 \pm 0.05$ & $14.54 \pm 0.05$ & $14.28 \pm 0.07$ \\
\hline 9 & $17.60 \pm 0.04$ & $17.61 \pm 0.02$ & $17.07 \pm 0.02$ & $16.75 \pm 0.03$ & $16.42 \pm 0.02$ & $15.97 \pm 0.09$ & $15.73 \pm 0.15$ & $15.25^{a}$ \\
\hline 10 & $19.48 \pm 0.10$ & $18.89 \pm 0.02$ & $18.02 \pm 0.02$ & $17.57 \pm 0.02$ & $17.14 \pm 0.02$ & - & - & - \\
\hline 11 & $15.21 \pm 0.04$ & $15.21 \pm 0.02$ & $14.71 \pm 0.02$ & $14.37 \pm 0.02$ & $14.03 \pm 0.02$ & - & - & - \\
\hline 12 & $16.33 \pm 0.05$ & $15.74 \pm 0.03$ & $14.87 \pm 0.02$ & $14.42 \pm 0.06$ & $14.00 \pm 0.05$ & $13.24 \pm 0.02$ & $12.82 \pm 0.03$ & $12.74 \pm 0.03$ \\
\hline 13 & $19.37 \pm 0.07$ & $18.77 \pm 0.01$ & $17.99 \pm 0.02$ & $17.52 \pm 0.03$ & $17.08 \pm 0.03$ & $16.35 \pm 0.13$ & $15.68 \pm 0.13$ & $15.93 \pm 0.29$ \\
\hline 14 & $16.83 \pm 0.04$ & $16.79 \pm 0.01$ & $16.24 \pm 0.03$ & $15.92 \pm 0.06$ & $15.57 \pm 0.02$ & $14.95 \pm 0.04$ & $14.73 \pm 0.06$ & $14.79 \pm 0.11$ \\
\hline 15 & $18.52 \pm 0.04$ & $17.84 \pm 0.01$ & $16.97 \pm 0.03$ & $16.46 \pm 0.03$ & $16.03 \pm 0.03$ & $15.26 \pm 0.05$ & $14.79 \pm 0.07$ & $14.70 \pm 0.10$ \\
\hline
\end{tabular}

Note. ${ }^{a}$ No value for the uncertainty is given by the 2-MASS catalogue.

Table B5. Sloan Vega magnitudes and associated errors for the stellar sequence used in the calibration process of SN 2011fu's photometry.

\begin{tabular}{lccccc}
\hline Star & $\begin{array}{c}u \\
(\mathrm{mag})\end{array}$ & $\begin{array}{c}g^{a} \\
(\mathrm{mag})\end{array}$ & $\begin{array}{c}r \\
(\mathrm{mag})\end{array}$ & $\begin{array}{c}i \\
(\mathrm{mag})\end{array}$ & $\begin{array}{c}z \\
(\mathrm{mag})\end{array}$ \\
\hline 1 & - & - & $17.20 \pm 0.01$ & $16.64 \pm 0.01$ & $16.28 \pm 0.01$ \\
2 & - & $17.38 \pm 0.01$ & $17.15 \pm 0.01$ & $16.77 \pm 0.01$ & $16.52 \pm 0.03$ \\
3 & - & $17.44 \pm 0.01$ & $17.22 \pm 0.01$ & $16.83 \pm 0.01$ & $16.59 \pm 0.01$ \\
4 & - & $17.19 \pm 0.01$ & $16.63 \pm 0.01$ & $16.13 \pm 0.01$ & $15.82 \pm 0.01$ \\
5 & $17.85 \pm 0.02$ & $17.04 \pm 0.01$ & $16.89 \pm 0.01$ & $16.54 \pm 0.01$ & $16.34 \pm 0.02$ \\
6 & $15.52 \pm 0.03$ & - & $14.45 \pm 0.01$ & $14.06 \pm 0.01$ & $13.83 \pm 0.01$ \\
7 & $17.59 \pm 0.05$ & $16.96 \pm 0.01$ & $16.75 \pm 0.01$ & $16.36 \pm 0.01$ & $16.12 \pm 0.01$ \\
8 & - & $17.73 \pm 0.01$ & $16.83 \pm 0.01$ & $16.16 \pm 0.01$ & $15.71 \pm 0.01$ \\
9 & $17.59 \pm 0.05$ & $16.88 \pm 0.01$ & $16.72 \pm 0.01$ & $16.38 \pm 0.01$ & $16.16 \pm 0.02$ \\
10 & - & $17.96 \pm 0.01$ & $17.57 \pm 0.01$ & $17.12 \pm 0.01$ & $16.84 \pm 0.03$ \\
11 & $15.05 \pm 0.02$ & - & $14.32 \pm 0.02$ & $13.98 \pm 0.02$ & $13.84 \pm 0.04$ \\
12 & $16.15 \pm 0.03$ & - & $14.38 \pm 0.04$ & $13.97 \pm 0.01$ & $13.68 \pm 0.01$ \\
13 & - & $17.87 \pm 0.01$ & $17.53 \pm 0.01$ & $17.05 \pm 0.01$ & $16.78 \pm 0.02$ \\
14 & - & - & $15.88 \pm 0.01$ & $15.52 \pm 0.01$ & $15.29 \pm 0.01$ \\
15 & - & $15.95 \pm 0.01$ & $16.46 \pm 0.01$ & $16.00 \pm 0.01$ & $15.71 \pm 0.01$ \\
\hline
\end{tabular}

Note. ${ }^{a}$ The stars were only measured in one epoch in this band.

This paper has been typeset from a $\mathrm{TE}_{\mathrm{E}} \mathrm{X} / \mathrm{L} \mathrm{T} \mathrm{E} X$ file prepared by the author. 